\title{
A More Efficient Computationally Sound Non-Interactive Zero-Knowledge Shuffle Argument*
}

\author{
Helger Lipmaa ${ }^{1}$ and Bingsheng Zhang ${ }^{2}$ \\ 1 University of Tartu, Estonia \\ 2 State University of New York at Buffalo, USA
}

\begin{abstract}
We propose a new non-interactive (perfect) zero-knowledge (NIZK) shuffle argument that, when compared the only previously known efficient NIZK shuffle argument by Groth and $\mathrm{Lu}$, has a small constant factor times smaller computation and communication, and is based on more standard computational assumptions. Differently from Groth and Lu who only prove the co-soundness of their argument under purely computational assumptions, we prove computational soundness under a necessary knowledge assumption. We also present a general transformation that results in a shuffle argument that has a quadratically smaller common reference string (CRS) and a small constant factor times times longer argument than the original shuffle.

Our main technical result is a "1-sparsity" argument that has linear CRS length and prover's communication. This should be compared to the basic arguments of Groth (Asiacrypt 2010) and Lipmaa (TCC 2012), where the prover's computational complexity is quadratic. This gives a new insight to the NIZK arguments of Groth and Lipmaa, and we hope that the 1-sparsity argument (and possible related future basic arguments) can be used to build NIZK arguments for other interesting languages.
\end{abstract}

Keywords. Bilinear pairings, cryptographic shuffle, non-interactive zero-knowledge, progressionfree sets.

\section{Introduction}

In a shuffle argument, the prover proves that two tuples of randomized ciphertexts encrypt the same multiset of plaintexts. Such an argument is needed in e-voting and anonymous broadcast. In the case of e-voting, shuffles are used to destroy the relation between the voters and their ballots. There, the voters first encrypt their ballots. The ciphertexts are then sequentially shuffled by several independent mix servers, where every server also produces a zero-knowledge GMR85] shuffle argument. At the end, all shuffle arguments are verified and the final ciphertexts are threshold-decrypted. If all arguments are accepted, then the shuffle is correct. Moreover, as long as one mix server is honest, the shuffle remains private (that is, one cannot relate the voters and their ballots). As a completely different application, we point out simulatable oblivious transfer [KNP10]KNP11, where the use of shuffle makes it possible for the client to query database elements by using permuted indexes.

A lot of research [Cha81|Nef01|FS01|Gro03] has been conducted in the area of constructing secure and efficient shuffle arguments, with recent work resulting in shuffles that have sublinear communication and very competitive computational complexity. However, it is also important that the shuffle argument is non-interactive, due to the fact that non-interactive arguments are transferable (create once, verify many times without interacting with the prover). This is especially important in e-voting, where the correctness of e-voting (and thus of the shuffle) should be verifiable in years to come. Practically all previous shuffle arguments are interactive, and can only be made non-interactive by using the FiatShamir heuristic [FS86, that is, in the random oracle model. For example, Groth and Ishai GI08, Groth [Gro09, and Bayer and Groth [BG12] have constructed shuffle arguments with communication $\Theta\left(n^{2 / 3}\right), \Theta\left(n^{1 / 2}\right)$, and $\Theta\left(n^{1 / 2}\right)$ respectively, where $n$ is the number of ciphertexts. Unfortunately, they make use of the Schwartz-Zippel lemma Sch80] that requires the verifier to first provide a random input. The only known way to make the Schwartz-Zippel lemma based arguments non-interactive is to use the random oracle model. Unfortunately, it is well-known [CGH98|GK03] that there are protocols that are secure in the random oracle model but not in the plain model. Even if there are no similar distinguishing

^ Full version corresponding to a paper published at SCN 2012 LZ12. First eprint version was published on July 21, 2011. This version is from June 30, 2012. 


\begin{tabular}{|c|c|c|c|c|c|c|c|}
\hline & |CRS|| & Comm. & $\mathcal{P}$ 's comp. & $\mathcal{V}$ 's comp. & Pairing & Sound & Assumption \\
\hline GL07 & $2 n+8$ & $15 n+120$ & $51 n+246$ & $75 n+282$ & Sym. & Co- & $\mathrm{PPA}+\mathrm{SPA}+\mathrm{DLIN}$ \\
\hline Sect. 5 & $7 n+6$ & $6 n+11$ & $17 n+16$ & $28 n+18$ & Asym. & Sound & PKE + PSDL + DLIN \\
\hline App. $|\bar{D}|$ & $7 \sqrt{n}+6$ & $0 n+33 \sqrt{n}$ & $n+48 \sqrt{n}$ & $34 n+54 \sqrt{n}$ & Asym. & Sound & PKE + PSDL + DLIN \\
\hline
\end{tabular}

Table 1. Brief comparison of existing (not random-oracle based) and new (two last ones) NIZK shuffle arguments. Here, the communication complexity and the CRS length are given in group elements, prover's computation is given in exponentiations, and verifier's computation is given in (symmetric or asymmetric) bilinear pairings

attacks against any of the existing shuffle arguments, it is prudent to design alternative non-interactive shuffle arguments that are not based on random oracle model.

The only known (not random-oracle based) efficient non-interactive zero-knowledge (NIZK) shuffle argument (for the BBS cryptosystem [BBS04]) was proposed by Groth and Lu in GL07. The security of the Groth-Lu argument is based on the common reference string model (since non-interactivity is strongly desired, one cannot use a weaker model like the bare public key model [SV12]) and on two new computational assumptions, the permutation pairing assumption (PPA, see App. C) and the simultaneous pairing assumption (SPA). While Groth and Lu proved that their assumptions are secure in the generic group model, one can argue that their assumptions are specifically constructed so as the concrete shuffle argument will be co-sound [GOS11] (see [GL07] and Sect.2 for discussions on co-soundness). It is therefore interesting to construct a shuffle argument from "more standard" assumptions. Moreover, their shuffle argument has a relatively large computational complexity and communication complexity. (See Tbl. 1 . for a comparison.)

Our Contributions. We construct a new non-interactive shuffle argument that has better communication and is based on more standard computational security assumptions than the Groth-Lu argument. Full comparison between the Groth-Lu and the new argument is given later. Recall that permutation matrix is a Boolean matrix that has exactly one 1 in every row and column. From a very high-level point of view, following [FS01] and subsequent papers, we let the prover to commit to a permutation matrix and then present an efficient permutation matrix argument (given commitments commit to a permutation matrix). We then prove that the plaintext vector corresponding to the output ciphertext vector is equal to the product of this matrix and the plaintext vector corresponding to the input ciphertext vector, and thus is correctly formed. Both parts are involved. In particular, coming up with a characterization of permutation matrices that allows for an efficient cryptographic implementation was not a trivial task.

Terelius and Wikström [TW10] constructed an interactive permutation matrix argument based on the fact that a matrix is a permutation matrix iff its every column sums to 1 and its every row has exactly one non-zero element. To verify that the committed matrix satisfies these properties, they used the Schwartz-Zippel lemma with the verifier sending a random vector to the prover. This introduces interaction (or the use of a random oracle). We do not know how to prove efficiently in NIZK that a commitment commits to a unit vector; how to construct such an efficient argument is an interesting open problem. We propose a superficially similar permutation matrix argument that is based on the (related) fact that a matrix is a permutation matrix exactly if every column sums to 1 and every row has at most one non-zero element. However, we do not explicitly use the Schwartz-Zippel lemma, and this makes it possible for us to create a NIZK argument without using the random oracle model.

Cryptographically, the new permutation matrix argument is based on recent techniques of Groth [Gro10 and Lipmaa Lip12 who proposed an NIZK argument for circuit satisfiability based on two subarguments, for Hadamard - that is, entry-wise - product and permutation. (The same basic arguments were then used in CLZ12 to construct an efficient non-interactive range proof.) Unfortunately, in their subarguments, the prover has quadratic (or quasilinear $O\left(n 2^{2 \sqrt{2 \log _{2} n}}\right.$ ), if one only counts the group operations) computational complexity. This is not acceptable in our case, and therefore we do not use any of the arguments that were constructed in Gro10 Lip12].

We propose 2 new basic arguments (a zero argument, see Sect. 3.1 and a 1-sparsity argument, see Sect. 3.2), and then combine them in Sect. 3.3 to form a permutation matrix argument. The zero 
argument (the prover can open the given commitment to the zero tuple) can be interpreted as a knowledge of the discrete logarithm argument, and is a special case of Groth's restriction argument from Gro10. On the other hand, the 1-sparsity argument (the prover can open the given commitment to a tuple $\boldsymbol{a}=\left(a_{1}, \ldots, a_{n}\right)$, where at most one coordinate $a_{i}$ is non-zero) is conceptually new.

Like the basic arguments of [Lip12], the new 1-sparsity argument relies on the existence of a dense progression-free set. However, the costs of the 1-sparsity argument do not depend explicitly on the size of the used progression-free sets. Briefly, in Lip12 and the new 1-sparsity argument, the discrete logarithm of the non-interactive argument is equal to the sum of two polynomials $F_{c o n}(x)$ and $F_{\pi}(x)$, where $x$ is the secret key. The first polynomial $F_{c o n}$ has exactly one monomial per constraint that a honest prover has to satisfy. The number of constraints is linear (for any $i, a_{i} \cdot b_{i}=c_{i}$ ) in [Lip12] and quadratic (for any two different coefficients $a_{i}$ and $a_{j}, a_{i} \cdot a_{j}=0$ ) in the new 1-sparsity argument. The second polynomial consists of monomials (a quasilinear number $O\left(n 2^{2 \sqrt{2 \log _{2} n}}\right)$ in Lip12 and a linear number in the new 1-sparsity argument) that have to be computed by a honest prover during the argument, and this is the main reason why both the CRS length and the prover's computational complexity are lower in the 1-sparsity argument compared to the arguments in Lip12. We find this to be an interesting result by itself, leading to an obvious question whether similar arguments (that have a superlinear number of constraints and a linear number of spurious monomials) can be used as an underlying engine to construct other interesting NIZK proofs.

In Sect. 5, we combine the permutation matrix argument with a knowledge version of the BBS BBS04] cryptosystem to obtain an efficient NIZK shuffle argument. Informally, by the KE assumption [Dam91, in the knowledge BBS cryptosystem (defined in Sect. 4) the ciphertext creator knows both the used plaintext and the randomizer. Since it is usually not required that the ciphertext creator also knows the randomizer, the knowledge BBS cryptosystem satisfies a stronger than usual version of plaintextawareness. While this version of plaintext-awareness has not been considered in the literature before, it is also satisfied by the Damgård's Elgamal cryptosystem from Dam91.

According to [AF07, only languages in $\mathbf{P}$ /poly can have direct black-box perfect NIZK arguments 1 Since all known constructions of NIZK arguments use direct black-box reductions, one can argue that the "natural" definition of soundness is not the right definition of soundness for perfect NIZK arguments, see [GL07] for more discussion. To overcome the impossibility results of [AF07, Groth and Lu GL07] proved co-soundness GL07GOS11] of their argument under purely computational assumptions.

Our subarguments (the zero argument, the 1-sparsity argument, and the permutation matrix argument) are not computationally sound since their languages are based on a perfectly hiding commitment scheme, see Sect. 3. Instead, we prove that these arguments satisfy a weak version of soundness Gro10 Lip12 under purely computational assumptions. We could use a similar definition of the weak soundness of the shuffle argument and prove that the new shuffle argument is (weakly) sound by using only standard computational assumptions. Instead (mostly since computational soundness is a considerably more standard security requirement), we prove computational soundness of the shuffle argument under a (known) knowledge assumption. This is also the reason why we need to use the knowledge BBS cryptosystem.

Apart from the knowledge assumption, the security of the new shuffle argument is based on the DLIN assumption BBS04 (which is required for the CPA-security of the BBS cryptosystem), and on the power symmetric discrete logarithm (PSDL, see Sect. 2) assumption from Lip12. The PSDL assumption is much more standard(-looking) than the SPA and PPA assumptions from [GL07.

Comparison with [GL07]. Tbl.11 provides a comparison between [GL07] and the new shuffle argument. Since it was not stated in GL07, we have calculated ourselves ${ }^{2}$ the computational complexity of the Groth-Lu argument. As seen from Tbl. 11 the new argument is computationally about 2.5 to 3 times more efficient and communication-wise about 2 times more efficient, if one just counts the number of exponentiations (in the case of the prover's computation), pairings (verifier's computation), or group

\footnotetext{
${ }^{1}$ It is not necessary to have a perfect NIZK argument for a shuffle (one could instead construct a computational NIZK proof), but the techniques of both GL07 and especially of the current paper are better suited to construct efficient perfect NIZK arguments. We leave it as an open question to construct a computational NIZK proof for shuffle with a comparable efficiency.

${ }^{2}$ Our calculations are based on the Groth-Sahai proofs GS08 that were published after the Groth-Lu shuffle argument. The calculations may be slightly imprecise.
} 
elements (communication). In addition, the new argument uses asymmetric pairings $\hat{e}: \mathbb{G}_{1} \times \mathbb{G}_{2} \rightarrow \mathbb{G}_{T}$, while GL07 uses symmetric pairings with $\mathbb{G}_{1}=\mathbb{G}_{2}$. This means in particular that the difference in efficiency is larger than seen from Tbl. 1 First, asymmetric pairings themselves are much more efficient than symmetric pairings. Second, if asymmetric pairings were used in the Groth-Lu shuffle, one would have to communicate two different versions (one in group $\mathbb{G}_{1}$ and another one in group $\mathbb{G}_{2}$ ) of some of the group elements.

The main drawback of the new shuffle argument is that its soundness relies additionally on a knowledge assumption. However, a non-standard assumption is necessary to achieve perfect zeroknowledge AF07. Differently from the random oracle assumption that is known to be false in general CGH98|GK03, knowledge assumptions are just known to be non-falsifiable and thus might be true for any practical purposes. (In comparison, the Groth-Lu argument was proven to be co-sound, which is a weaker version of computational soundness, under purely computational assumptions.)

Moreover, the Groth-Lu shuffle uses the BBS cryptosystem (where one ciphertext is 3 group elements), while we use the new knowledge BBS cryptosystem (6 group elements). This difference is small compared to the reduction in the argument size. The use of knowledge BBS cryptosystem corresponds to adding a proof of knowledge of the plaintexts (and the randomizers) by the voters. However, it means that in the proof of soundness, we show security only against (white-box) adversaries who have access to the secret coins of all voters and mixservers. It is a reasonable compromise, comparable to the case in interactive (or Fiat-Shamir heuristic based) shuffles where the ballots are accompanied by a proof of knowledge of the ballot, from which either the adversary of the simulator can obtain the actual votes, but without the use of a random oracle, see Sect. 5 for more discussion. As we note there, our soundness definition follows that of [GL07, but the mentioned issues are due to the use of a knowledge assumption. We hope that the current work will motivate more research on clarifying such issues.

Another drawback of our scheme as compared to GL07 is that it uses a lifted cryptosystem, and thus can be only used to shuffle small plaintexts. This is fine in applications like e-voting (where the plaintext is a candidate number). Many of the existing e-voting schemes (for example, [CGS97]) are based on (lifted) Elgamal and thus require the plaintexts to be small. We note that significant speedups can be achieved in both cases by using efficient multi-exponentiation algorithms and thus for a meaningful computational comparison, one should implement the shuffle arguments.

Shorter CRS. In App. D, we show that one can transform both the Groth-Lu argument and the new argument, by using the Clos network [Clo53|DT04, to have a CRS of size $\Theta(\sqrt{n})$ while increasing the communication and computation by a small constant factor. This version of the new argument is computationally/communication-wise only slightly less efficient than the Groth-Lu argument but has a quadratically smaller CRS, see Tbl. 1. This transformation can be applied to any shuffle argument that has linear communication and computation, and a CRS of length $f(n)=\Omega(1)$. We pose it as an open problem to construct (may be using similar techniques) an NIZK shuffle argument where both the CRS and the communication are sublinear.

\section{Preliminaries}

Notation. Let $[n]=\{1,2, \ldots, n\}$. If $y=h^{x}$, then let $\log _{h} y:=x$. To help readability in cases like $g_{2}^{r_{i}+x^{\lambda} \psi^{-1}(i)}$, we sometimes write $\exp (h, x)$ instead of $h^{x}$. Let $\kappa$ be the security parameter. PPT denotes probabilistic polynomial time. For a tuple of integers $\Lambda=\left(\lambda_{1}, \ldots, \lambda_{n}\right)$ with $\lambda_{i}<\lambda_{i+1}$, let $\left(a_{i}\right)_{i \in \Lambda}=$ $\left(a_{\lambda_{1}}, \ldots, a_{\lambda_{n}}\right)$. We sometimes denote $\left(a_{i}\right)_{i \in[n]}$ as $\boldsymbol{a}$. We say that $\Lambda=\left(\lambda_{1}, \ldots, \lambda_{n}\right) \subset \mathbb{Z}$ is an $(n, \kappa)$-nice tuple, if $0<\lambda_{1}<\cdots<\lambda_{i}<\cdots<\lambda_{n}=\operatorname{poly}(\kappa)$. Let $S_{n}$ be the set of permutations from $[n]$ to $[n]$.

Additive combinatorics. By using notation that is common in additive combinatorics [TV06, if $\Lambda_{1}$ and $\Lambda_{2}$ are subsets of some additive group ( $\mathbb{Z}$ or $\mathbb{Z}_{p}$ within this paper), then $\Lambda_{1}+\Lambda_{2}=$ $\left\{\lambda_{1}+\lambda_{2}: \lambda_{1} \in \Lambda_{1} \wedge \lambda_{2} \in \Lambda_{2}\right\}$ is their sum set and $\Lambda_{1}-\Lambda_{2}=\left\{\lambda_{1}-\lambda_{2}: \lambda_{1} \in \Lambda_{1} \wedge \lambda_{2} \in \Lambda_{2}\right\}$ is their difference set. In particular, if $\Lambda$ is a set, then $k \Lambda=\left\{\sum_{i=1}^{k} \lambda_{i}: \lambda_{i} \in \Lambda\right\}$ is an iterated sumset. On the other hand, $k \cdot \Lambda=\{k \lambda: \lambda \in \Lambda\}$ is a dilation of $\Lambda$. We also let $2 \Lambda=\left\{\lambda_{1}+\lambda_{2}: \lambda_{1} \in \Lambda \wedge \lambda_{2} \in \Lambda \wedge \lambda_{1} \neq \lambda_{2}\right\} \subseteq \Lambda+\Lambda$ to denote a restricted sumset. 
Progression-Free Sets. A set $\Lambda=\left\{\lambda_{1}, \ldots, \lambda_{n}\right\}$ of integers is progression-free [TV06, if no three elements of $\Lambda$ are in arithmetic progression, that is, $\lambda_{i}+\lambda_{j}=2 \lambda_{k}$ only if $i=j=k$. Let $r_{3}(N)$ denote the cardinality of the largest progression-free set that belongs to $[N]$. Recently, Elkin Elk11] showed that

$$
r_{3}(N)=\Omega\left(\left(N \cdot \log ^{1 / 4} N\right) / 2^{2 \sqrt{2 \log _{2} N}}\right) .
$$

On the other hand, it is known that $r_{3}(N)=O\left(N(\log \log N)^{5} / \log N\right)$ San11]. Thus, according to [San11], the minimal $N$ such that $r_{3}(N)=n$ is $\omega(n)$, while according to Elkin, $N=O\left(n 2^{2 \sqrt{2 \log _{2} n}}\right)=n^{1+o(1)}$. Thus, for any fixed $n>0$, there exists $N=n^{1+o(1)}$, such that $[N]$ contains an $n$-element progression-free subset Lip12.

While the efficiency of arguments from Lip12 directly depended on the choice of the progression-free set, in our case the only thing dependent on this choice is the tightness of most of our security reductions; see the definition of PSDL below, or the proofs of Thm. 2, Thm. 4 and Thm. 5 . Due to this, one may opt to use a less dense (but easy to construct) progression-free set. As an example, Erdős and Turán ET36. defined a set $T(n)$ of all integers up to $n$ that have no number 2 in their ternary presentation. Clearly, $|T(n)| \approx n^{\log _{3} 2} \approx n^{0.63}$ and $T(n)$ is progression-free. One can obtain a dense set of progression-free odd positive integers by mapping every $a$ in $T(n)$ to $2 a+1$.

Bilinear groups. A bilinear group generator $\mathcal{G}_{\mathrm{bp}}\left(1^{\kappa}\right)$ outputs gk $:=\left(p, \mathbb{G}_{1}, \mathbb{G}_{2}, \mathbb{G}_{T}, \hat{e}, g_{1}, g_{2}\right) \leftarrow \mathcal{G}_{\mathrm{bp}}\left(1^{\kappa}\right)$ such that $p$ is a $\kappa$-bit prime, $\mathbb{G}_{1}, \mathbb{G}_{2}$ and $\mathbb{G}_{T}$ are multiplicative cyclic groups of order $p, \hat{e}: \mathbb{G}_{1} \times \mathbb{G}_{2} \rightarrow \mathbb{G}_{T}$ is a bilinear map (pairing), and $g_{t} \leftarrow \mathbb{G}_{t} \backslash\{1\}$ is a random generator of $\mathbb{G}_{t}$ for $t \in\{1,2\}$. Additionally, it is required that (a) $\forall a, b \in \mathbb{Z}, \hat{e}\left(g_{1}^{a}, g_{2}^{b}\right)=\hat{e}\left(g_{1}, g_{2}\right)^{a b}$, (b) $\hat{e}\left(g_{1}, g_{2}\right)$ generates $\mathbb{G}_{T}$, and (c) it is efficient to decide the membership in $\mathbb{G}_{1}, \mathbb{G}_{2}$ and $\mathbb{G}_{T}$, the group operations and the pairing $\hat{e}$ are efficiently computable, generators of $\mathbb{G}_{1}$ and $\mathbb{G}_{2}$ are efficiently sampleable, and the descriptions of the groups and group elements each are $O(\kappa)$ bit long. One can represent an element of $\mathbb{G}_{1} / \mathbb{G}_{2} / \mathbb{G}_{T}$ in respectively $512 / 256 / 3072$ bits, by using an optimal (asymmetric) Ate pairing HSV06 over a subclass of BarretoNaehrig curves BN05 PSNB11].

Public-key cryptosystem. A public-key cryptosystem $\left(\mathcal{G}_{\mathrm{bp}}, \mathcal{G}_{\mathrm{pkc}}, \mathcal{E}\right.$ nc, $\left.\mathcal{D e c}\right)$ is a tuple of efficient algorithms, where $\mathcal{G}_{\mathrm{bp}}$ is a bilinear group generator that outputs gk, $\mathcal{G}_{\mathrm{pkc}}(\mathrm{gk})$ generates a secret/public key pair (sk, pk), randomized encryption algorithm $\mathcal{E}_{\mathrm{nc}} \mathrm{pk}(\mu ; r)$ produces a ciphertext $c$, and deterministic decryption algorithm $\mathcal{D e c}_{\mathrm{sk}}(c)$ produces a plaintext $\mu$. It is required that for all gk $\leftarrow \mathcal{G}_{\mathrm{bp}}\left(1^{\kappa}\right)$, (sk, pk) $\in \mathcal{G}_{\mathrm{pkc}}(\mathrm{gk})$ and for all valid $\mu$ and $r, \mathcal{D e c}_{\mathrm{sk}}\left(\mathcal{E}_{\mathrm{nc}} \mathrm{pk}(\mu ; r)\right)=\mu$. Assume that the randomizer space $\mathcal{R}$ is efficiently sampleable. A public-key cryptosystem $\left(\mathcal{G}_{\mathrm{bp}}, \mathcal{G}_{\mathrm{pkc}}, \mathcal{E} \mathrm{nc}, \mathcal{D}\right.$ ec $)$ is $C P A$-secure, if for all stateful non-uniform PPT adversaries $\mathcal{A}$, the following probability is negligible in $\kappa$ :

$$
\left|\operatorname{Pr}\left[\begin{array}{l}
\mathrm{gk} \leftarrow \mathcal{G}_{\mathrm{bp}}\left(1^{\kappa}\right),(\mathrm{sk}, \mathrm{pk}) \leftarrow \mathcal{G}_{\mathrm{pkc}}(\mathrm{gk}),\left(\mu_{0}, \mu_{1}\right) \leftarrow \mathcal{A}(\mathrm{pk}), \\
b \leftarrow\{0,1\}, r \leftarrow \mathcal{R}: \mathcal{A}\left(\mathcal{E}_{\mathrm{nc}} \mathrm{pk}\left(\mu_{b} ; r\right)\right)=b
\end{array}\right]-\frac{1}{2}\right| .
$$

$\Lambda$-Power Symmetric Discrete Logarithm Assumption. Let $\Lambda$ be an $(n, \kappa)$-nice tuple for $n=$ poly $(\kappa)$. A bilinear group generator $\mathcal{G}_{\mathrm{bp}}$ is $\Lambda$-PSDL secure Lip12, if for any non-uniform PPT adversary $\mathcal{A}$

$$
\operatorname{Pr}\left[\mathrm{gk}:=\left(p, \mathbb{G}_{1}, \mathbb{G}_{2}, \mathbb{G}_{T}, \hat{e}, g_{1}, g_{2}\right) \leftarrow \mathcal{G}_{\mathrm{bp}}\left(1^{\kappa}\right), x \leftarrow \mathbb{Z}_{p}: \mathcal{A}\left(\mathrm{gk} ;\left(g_{1}^{x^{\ell}}, g_{2}^{x^{\ell}}\right)_{\ell \in \Lambda}\right)=x\right]
$$

is negligible in $\kappa$. (Note that $\mathcal{A}$ also has access to $g_{t}^{x^{0}}$ since it belongs to gk.) A version of PSDL assumption in a non pairing-based group was defined in GJM02. Lipmaa Lip12 proved that the $\Lambda$ PSDL assumption holds in the generic group model for any $(n, \kappa)$-nice tuple $\Lambda$ given that $n=\operatorname{poly}(\kappa)$. More precisely, any successful generic adversary for $\Lambda$-PSDL requires time $\Omega\left(\sqrt{p / \lambda_{n}}\right)$ where $\lambda_{n}$ is the largest element of $\Lambda$. Thus, the choice of the actual security parameter depends on $\lambda_{n}$ and thus also on $\Lambda$.

Non-Interactive Zero-Knowledge for Group-Specific Languages. Let $\mathcal{G}_{\mathrm{bp}}$ be a bilinear group generator, and let gk $:=\left(p, \mathbb{G}_{1}, \mathbb{G}_{2}, \mathbb{G}_{T}, \hat{e}, g_{1}, g_{2}\right) \leftarrow \mathcal{G}_{\mathrm{bp}}\left(1^{\kappa}\right)$. Let $R=\{(\mathrm{gk} ; C, w)\}$ be an efficiently computable group-specific binary relation such that $|w|=\operatorname{poly}(|C|)$. Here, $C$ is a statement, and $w$ is a 
witness. Let $L=\{(\mathrm{gk} ; C):(\exists w)(\mathrm{gk} ; C, w) \in R\}$ be a group-specific NP-language. Shuffle (see Sect. 5) has a natural corresponding group-specific language, since one proves a relation between elements of the same group.

A non-interactive argument for $R$ consists of the following PPT algorithms: a bilinear group generator $\mathcal{G}_{\text {bp }}$, a common reference string $(\mathrm{CRS})$ generator $\mathcal{G}_{\text {crs }}$, a prover $\mathcal{P}$, and a verifier $\mathcal{V}$. For gk $\leftarrow \mathcal{G}_{\text {bp }}\left(1^{\kappa}\right)$ and crs $\leftarrow \mathcal{G}_{\mathrm{crs}}(\mathrm{gk}), \mathcal{P}(\mathrm{gk}, \mathrm{crs} ; C, w)$ produces an argument $\pi$. The verifier $\mathcal{V}$ (gk, crs; $\left.C, \pi\right)$ outputs either 1 (accept) or 0 (reject). If the verifier only accesses a small part $\mathrm{crs}_{v}$ of crs, we say that $\mathrm{crs}_{v}$ is the verifier's part of the CRS and we will give just $\mathrm{crs}_{v}$ as an input to $\mathcal{V}$. When efficiency is not important (e.g., in the security definitions), we give the entire crs to $\mathcal{V}$.

An argument $\left(\mathcal{G}_{\mathrm{bp}}, \mathcal{G}_{\mathrm{crs}}, \mathcal{P}, \mathcal{V}\right)$ is perfectly complete, if for all gk $\leftarrow \mathcal{G}_{\mathrm{bp}}\left(1^{\kappa}\right)$, all crs $\leftarrow \mathcal{G}_{\mathrm{crs}}(\mathrm{gk})$ and all $(C, w)$ such that $(\mathrm{gk} ; C, w) \in R, \mathcal{V}(\mathrm{gk}, \mathrm{crs} ; C, \mathcal{P}(\mathrm{gk}, \mathrm{crs} ; C, w))=1$. An argument $\left(\mathcal{G}_{\mathrm{bp}}, \mathcal{G}_{\mathrm{crs}}, \mathcal{P}, \mathcal{V}\right)$ is adaptively computationally sound, if for all non-uniform PPT adversaries $\mathcal{A}$, the probability $\operatorname{Pr}[\mathrm{gk} \leftarrow$ $\left.\mathcal{G}_{\mathrm{bp}}\left(1^{\kappa}\right), \mathrm{crs} \leftarrow \mathcal{G}_{\mathrm{crs}}(\mathrm{gk}),(C, \pi) \leftarrow \mathcal{A}(\mathrm{gk}, \mathrm{crs}):(\mathrm{gk} ; C) \notin L \wedge \mathcal{V}(\mathrm{gk}, \mathrm{crs} ; C, \pi)=1\right]$ is negligible in $\kappa$ The soundness is adaptive in the sense that the adversary sees the CRS before producing the statement $C$. An argument $\left(\mathcal{G}_{\mathrm{bp}}, \mathcal{G}_{\mathrm{crs}}, \mathcal{P}, \mathcal{V}\right)$ is perfectly witness-indistinguishable, if for all gk $\in \mathcal{G}_{\mathrm{bp}}\left(1^{\kappa}\right)$, crs $\in \mathcal{G}_{\text {crs }}(\mathrm{gk})$ and $\left(\left(\mathrm{gk} ; C, w_{0}\right),\left(\mathrm{gk} ; C, w_{1}\right)\right) \in R^{2}$, the distributions $\mathcal{P}\left(\mathrm{gk}, \mathrm{crs} ; C, w_{0}\right)$ and $\mathcal{P}\left(\mathrm{gk}, \mathrm{crs} ; C, w_{1}\right)$ are equal. An argument $\left(\mathcal{G}_{\mathrm{bp}}, \mathcal{G}_{\mathrm{crs}}, \mathcal{P}, \mathcal{V}\right)$ is perfectly zero-knowledge, if there exists a PPT simulator $\mathcal{S}=\left(\mathcal{S}_{1}, \mathcal{S}_{2}\right)$, such that for all stateful interactive non-uniform PPT adversaries $\mathcal{A}, \quad \operatorname{Pr}[\mathrm{gk} \leftarrow$ $\left.\mathcal{G}_{\mathrm{bp}}\left(1^{\kappa}\right), \mathrm{crs} \leftarrow \mathcal{G}_{\mathrm{crs}}(\mathrm{gk}),(C, w) \leftarrow \mathcal{A}(\mathrm{gk}, \mathrm{crs}), \pi \leftarrow \mathcal{P}(\mathrm{gk}, \mathrm{crs} ; C, w):(\mathrm{gk} ; C, w) \in R \wedge \mathcal{A}(\pi)=1\right]=\operatorname{Pr}[\mathrm{gk} \leftarrow$ $\left.\mathcal{G}_{\mathrm{bp}}\left(1^{\kappa}\right),(\mathrm{crs}, \mathrm{td}) \leftarrow \mathcal{S}_{1}(\mathrm{gk}),(C, w) \leftarrow \mathcal{A}(\mathrm{gk}, \mathrm{crs}), \pi \leftarrow \mathcal{S}_{2}(\mathrm{gk}, \mathrm{crs}, \mathrm{td} ; C):(\mathrm{gk} ; C, w) \in R \wedge \mathcal{A}(\pi)=1\right]$. Here, td is the simulation trapdoor.

$\Lambda$-Power Knowledge of Exponent Assumption ( $\boldsymbol{\Lambda}$-PKE). The soundness of NIZK arguments (for example, an argument that a computationally binding commitment scheme commits to 0) seems to be an unfalsifiable assumption in general. We will use a weaker version of soundness in the subarguments, but in the case of the shuffle argument, we will prove soundness. Similarly to Gro10Lip12, we will base the soundness of that argument on an explicit knowledge assumption.

For two algorithms $\mathcal{A}$ and $X_{\mathcal{A}}$, we write $(y ; z) \leftarrow\left(\mathcal{A} \| X_{\mathcal{A}}\right)(x)$ if $\mathcal{A}$ on input $x$ outputs $y$, and $X_{\mathcal{A}}$ on the same input (including the random tape of $\mathcal{A}$ ) outputs $z$. Let $\Lambda$ be an $(n, \kappa)$-nice tuple for some $n=\operatorname{poly}(\kappa)$. Consider $t \in\{1,2\}$. The bilinear group generator $\mathcal{G}_{\mathrm{bp}}$ is $\Lambda$-PKE secure in group $\mathbb{G}_{t}$ if for any non-uniform PPT adversary $\mathcal{A}$ there exists a non-uniform PPT extractor $X_{\mathcal{A}}$, such that

$$
\operatorname{Pr}\left[\begin{array}{l}
\text { gk }:=\left(p, \mathbb{G}_{1}, \mathbb{G}_{2}, \mathbb{G}_{T}, \hat{e}, g_{1}, g_{2}\right) \leftarrow \mathcal{G}_{\mathrm{bp}}\left(1^{\kappa}\right),(\alpha, x) \leftarrow \mathbb{Z}_{p}^{2}, \\
\operatorname{crs} \leftarrow\left(g_{t}^{\alpha},\left(g_{t}^{x^{\ell}}, g_{t}^{\alpha x^{\ell}}\right)_{\ell \in \Lambda}\right),\left(c, \hat{c} ;\left(a_{\ell}\right)_{\ell \in\{0\} \cup \Lambda}\right) \leftarrow\left(\mathcal{A} \| X_{\mathcal{A}}\right)(\mathrm{gk} ; \mathrm{crs}): \\
\hat{c}=c^{\alpha} \wedge c \neq \prod_{\ell \in\{0\} \cup \Lambda} g_{t}^{a_{\ell} x^{\ell}}
\end{array}\right]
$$

is negligible in $\kappa$. Note that the element $a_{0}$ is output since $g_{t}$ belongs to the CRS, and thus the adversary has access to $\left(g_{t}^{x^{\ell}}, g_{t}^{\alpha x^{\ell}}\right)$ for $\ell \in\{0\} \cup \Lambda$. Groth Gro10 proved that the $\Lambda$-PKE assumption holds in the generic group model in the case $\Lambda=[n]$; his proof can be straightforwardly modified to the general case. We later need the special case where $\Lambda=\emptyset$, that is, the CRS contains only $g_{t}^{\alpha}$, and the extractor returns $a_{0}$ such that $c=g_{t}^{a_{0}}$. This KE assumption (in a bilinear group) is similar to Damgård's KE assumption [Dam91, except that it is made in a bilinear group setting.

Commitment Schemes in the CRS Model. A (tuple) commitment scheme $\left(\mathcal{G}_{\text {com }}, \mathcal{C}\right.$ om $)$ consists of two PPT algorithms: a randomized CRS generation algorithm $\mathcal{G}_{\text {com }}$, and a randomized commitment algorithm $\mathcal{C}$ om. Here, $\mathcal{G}_{\text {com }}^{t}\left(1^{\kappa}, n\right), t \in\{1,2\}$, produces a CRS ck $t$, and $\mathcal{C}_{\mathrm{Com}^{t}}\left(\mathrm{ck}_{t} ; \boldsymbol{a} ; r\right)$, with $\boldsymbol{a}=\left(a_{1}, \ldots, a_{n}\right)$, outputs a commitment value $A \in \mathbb{G}_{t}$. Within this paper, we open a commitment $\mathcal{C o m}^{t}\left(\mathrm{ck}_{t} ; \boldsymbol{a} ; r\right)$ by publishing the values $\boldsymbol{a}$ and $r$.

A commitment scheme $\left(\mathcal{G}_{\text {com }}, \mathcal{C}\right.$ om $)$ is computationally binding in group $\mathbb{G}_{t}$, if for every non-uniform PPT adversary $\mathcal{A}$ and positive integer $n=\operatorname{poly}(\kappa)$, the probability

$$
\operatorname{Pr}\left[\begin{array}{l}
\mathrm{ck}_{t} \leftarrow \mathcal{G}_{\mathrm{com}}^{t}\left(1^{\kappa}, n\right),\left(\boldsymbol{a}_{\mathbf{1}}, r_{1}, \boldsymbol{a}_{\mathbf{2}}, r_{2}\right) \leftarrow \mathcal{A}\left(\mathrm{ck}_{t}\right): \\
\left(\boldsymbol{a}_{\mathbf{1}}, r_{1}\right) \neq\left(\boldsymbol{a}_{\mathbf{2}}, r_{2}\right) \wedge \mathcal{C O m}^{t}\left(\mathrm{ck}_{t} ; \boldsymbol{a}_{\mathbf{1}} ; r_{1}\right)=\mathcal{C} \operatorname{Com}^{t}\left(\mathrm{ck}_{t} ; \boldsymbol{a}_{\mathbf{2}} ; r_{2}\right)
\end{array}\right]
$$


is negligible in $\kappa$. A commitment scheme $\left(\mathcal{G}_{\text {com }}, \mathcal{C}\right.$ om $)$ is perfectly hiding in group $\mathbb{G}_{t}$, if for any positive integer $n=\operatorname{poly}(\kappa)$ and $\mathrm{ck}_{t} \in \mathcal{G}_{\text {com }}^{t}\left(1^{\kappa}, n\right)$ and any two messages $\boldsymbol{a}_{\mathbf{1}}$ and $\boldsymbol{a}_{\mathbf{2}}$, the distributions $\mathcal{C o m}^{t}\left(\mathrm{ck}_{t} ; \boldsymbol{a}_{\mathbf{1}} ; \cdot\right)$ and $\mathcal{C o m}^{t}\left(\mathrm{ck}_{t} ; \boldsymbol{a}_{\mathbf{2}} ; \cdot\right)$ are equal. We use the following variant of the knowledge commitment scheme from [Gro10] as modified by Lipmaa Lip12:

CRS generation $\mathcal{G}_{\text {com }}^{t}\left(1^{\kappa}, n\right)$ : Let $\Lambda$ be an $(n, \kappa)$-nice tuple with $n=\operatorname{poly}(\kappa)$. Define $\lambda_{0}=0$. Given a bilinear group generator $\mathcal{G}_{\mathrm{bp}}$, set gk $:=\left(p, \mathbb{G}_{1}, \mathbb{G}_{2}, \mathbb{G}_{T}, \hat{e}, g_{1}, g_{2}\right) \leftarrow \mathcal{G}_{\mathrm{bp}}\left(1^{\kappa}\right)$. Choose random $\alpha, x \leftarrow \mathbb{Z}_{p}$. The CRS is $\mathrm{ck}_{t} \leftarrow\left(\mathrm{gk} ; \hat{g}_{t},\left(g_{t i}, \hat{g}_{t i}\right)_{i \in[n]}\right)$, where $g_{t i}=g_{t}^{x^{\lambda_{i}}}$ and $\hat{g}_{t i}=g_{t}^{\alpha x^{\lambda_{i}}}$. Note that $g_{t}=g_{t 0}$ is a part of gk.

Commitment: To commit to $\boldsymbol{a}=\left(a_{1}, \ldots, a_{n}\right) \in \mathbb{Z}_{p}^{n}$ in group $\mathbb{G}_{t}$, the committing party chooses a random $r \leftarrow \mathbb{Z}_{p}$, and defines $\mathcal{C o m}^{t}\left(\mathrm{ck}_{t} ; \boldsymbol{a} ; r\right):=\left(g_{t}^{r} \cdot \prod_{i=1}^{n} g_{t i}^{a_{i}}, \hat{g}_{t}^{r} \cdot \prod_{i=1}^{n} \hat{g}_{t i}^{a_{i}}\right)$.

Let $t=1$. Fix a commitment key ck k $_{1}$ that in particular specifies $g_{2}, \hat{g}_{2} \in \mathbb{G}_{2}$. A commitment $(A, \hat{A}) \in \mathbb{G}_{1}^{2}$ is valid, if $e\left(A, \hat{g}_{2}\right)=e\left(\hat{A}, g_{2}\right)$. The case of $t=2$ is dual.

As shown in [Lip12, the knowledge commitment scheme in group $\mathbb{G}_{t}$ is perfectly hiding, and computationally binding under the $\Lambda$-PSDL assumption in group $\mathbb{G}_{t}$. If the $\Lambda$-PKE assumption holds in group $\mathbb{G}_{t}$, then for any non-uniform PPT algorithm $\mathcal{A}$, that outputs some valid knowledge commitments there exists a non-uniform PPT extractor $X_{\mathcal{A}}$ that, given as an input the input of $\mathcal{A}$ together with $\mathcal{A}$ 's random coins, extracts the contents of these commitments.

A trapdoor commitment scheme has 3 additional efficient algorithms: (a) A trapdoor CRS generation algorithm inputs $t, n$ and $1^{\kappa}$ and outputs a CRS $\mathrm{ck}^{*}$ (that has the same distribution as $\mathcal{G}_{\mathrm{com}}^{t}\left(1^{\kappa}, n\right)$ ) and a trapdoor $\mathrm{td}$, (b) a randomized trapdoor commitment that takes $\mathrm{ck}^{*}$ and a randomizer $r$ as inputs and outputs the value $\mathcal{C o m}^{t}\left(\mathrm{ck}^{*} ; \mathbf{0} ; r\right)$, and (c) a trapdoor opening algorithm that takes $\mathrm{ck}^{*}, \mathrm{td}, \boldsymbol{a}$ and $r$ as an input and outputs an $r^{\prime}$, s.t. $\mathcal{C o m}^{t}\left(\mathrm{ck}^{*} ; \mathbf{0} ; r\right)=\mathcal{C}_{\mathrm{om}^{t}}\left(\mathrm{ck}^{*} ; \boldsymbol{a} ; r^{\prime}\right)$. The knowledge commitment scheme is trapdoor, with the trapdoor being td $=x$ : after trapdoor-committing $A \leftarrow \mathcal{C o m}^{t}(\mathrm{ck} ; \mathbf{0} ; r)=g_{t}^{r}$ for $r \leftarrow \mathbb{Z}_{p}$, the committer can open it to $\left(\boldsymbol{a} ; r-\sum_{i=1}^{n} a_{i} x^{\lambda_{i}}\right)$ for any $\boldsymbol{a}$ Gro10 [Lip12.

Adaptive $\boldsymbol{R}_{\boldsymbol{c o}}$-Soundness. To avoid knowledge assumptions, one can relax the notion of soundness. Following GOS11] and GL07, $R_{c o}$-soundness is a weaker version of soundness, where it is required that an adversary who knows that $(\mathrm{gk} ; C) \notin L$ should not be able to produce a witness $w_{c o}$ such that (gk; $\left.C, w_{c o}\right) \in R_{c o}$ (see [GL07] or GOS11] for a longer explanation). More formally, let $R=\{(\mathrm{gk} ; C, w)\}$ and $L=\{(\mathrm{gk} ; C):(\exists w)(\mathrm{gk} ; C, w) \in R\}$ be defined as earlier. Let $R_{c o}=\left\{\left(\mathrm{gk} ; C, w_{c o}\right)\right\}$ be an efficiently computable binary relation. An argument $\left(\mathcal{G}_{\mathrm{bp}}, \mathcal{G}_{\mathrm{crs}}, \mathcal{P}, \mathcal{V}\right)$ is (adaptively) $R_{\text {co }}$-sound, if for all non-uniform $\mathrm{PPT}$ adversaries $\mathcal{A}$, the following probability is negligible in $\kappa$ :

$$
\operatorname{Pr}\left[\begin{array}{l}
\mathrm{gk} \leftarrow \mathcal{G}_{\mathrm{bp}}\left(1^{\kappa}\right), \mathrm{crs} \leftarrow \mathcal{G}_{\mathrm{crs}}(\mathrm{gk}),\left(C, w_{\mathrm{co}}, \pi\right) \leftarrow \mathcal{A}(\mathrm{gk}, \mathrm{crs}): \\
\left(\mathrm{gk} ; C, w_{\mathrm{co}}\right) \in R_{c o} \wedge \mathcal{V}(\mathrm{gk}, \mathrm{crs} ; C, \pi)=1
\end{array}\right] .
$$

Groth-Lipmaa Sublinear NIZK Arguments. In Gro10, Groth proposed efficient NIZK arguments that he proved to be sound under the power computational Diffie-Hellman assumption and the PKE assumption. Groth's arguments were later made more efficient by Lipmaa [Lip12, who also showed that one can use somewhat weaker security assumptions (PSDL instead of PCDH). Groth Gro10 and Lipmaa Lip12 proposed two basic arguments (for Hadamard product and permutation). In both cases, Lipmaa showed that by using results about progression-free sets one can construct a set $\Lambda_{2}$ with $\left|\Lambda_{2}\right|=O\left(n 2^{2 \sqrt{2 \log _{2} n}}\right)=n^{1+o(1)}$. Together with a trivial Hadamard sum argument, one obtains a complete set of arguments that can be used to construct NIZK arguments for any NP language. (See Gro10Lip12 for discussion.) However, this is always not the most efficient way to obtain a NIZK argument for a concrete language. In Sect. 3 we define new basic arguments that enable us to construct a very efficient permutation matrix argument and thus also a very efficient shuffle argument.

\section{New Subarguments}

In this section we present some subarguments that are required to construct the final shuffle argument. However, we expect them to have independent applications and thus we will handle each of them separately. 
CRS generation $\mathcal{G}_{\mathrm{crs}}\left(1^{\kappa}\right)$ : Let gk $:=\left(p, \mathbb{G}_{1}, \mathbb{G}_{2}, \mathbb{G}_{T}, \hat{e}, g_{1}, g_{2}\right) \leftarrow \mathcal{G}_{\mathrm{bp}}\left(1^{\kappa}\right)$. Let $\stackrel{\circ}{\alpha} \leftarrow \mathbb{Z}_{p}$. Denote $\stackrel{\circ}{g}_{t} \leftarrow g_{t}^{\alpha}$ for $t \in\{1,2\}$. The CRS is crs $\leftarrow\left(\stackrel{\circ}{g}_{1}, \stackrel{\circ}{g}_{2}\right)$. The commitment key is $\mathrm{ck}_{2} \leftarrow\left(\mathrm{gk} ; \stackrel{\circ}{g}_{2}\right)$, and the verifier's part of the CRS is $\operatorname{crs}_{v} \leftarrow \stackrel{\circ}{g}_{1}$.

Common input: $A_{2} \leftarrow g_{2}^{r} \in \mathbb{G}_{2}$.

Argument generation $\mathcal{P}_{0}\left(\mathrm{gk}, \mathrm{crs} ; A_{2}, r\right)$ : The prover defines $\stackrel{\circ}{2}_{2} \leftarrow \stackrel{\circ}{g}_{2}^{r}$, and sends $\pi \leftarrow \AA_{2} \in \mathbb{G}_{2}$ to $\mathcal{V}$ as the argument.

Verification $\mathcal{V}_{0}\left(\mathrm{gk}, \mathrm{crs}_{v} ; A_{2}, \pi=\AA_{2}\right)$ : The verifier accepts if $\hat{e}\left(\stackrel{\circ}{g}_{1}, A_{2}\right)=\hat{e}\left(g_{1}, \AA_{2}\right)$.

Protocol 1: New zero argument in group $\mathbb{G}_{2}$

\subsection{New Zero Argument}

In a zero argument, the prover aims to convince the verifier that he knows how to open knowledge commitment $A_{t} \in \mathbb{G}_{t}$ to the all-zero message tuple $\mathbf{0}=(0, \ldots, 0)$. Alternatively, one aims to prove the knowledge of the discrete logarithm of $A_{t}$, that is, that $A_{t}=g_{t}^{r}$ for some $r$. By using the homomorphic properties of the knowledge commitment scheme, the prover can use the zero argument to show that $A_{t}$ can be opened to an arbitrary constant.

This argument can be derived from Gro10Lip12. Intuitively, we set (only for this argument) $n=0$ and show that $A=A_{2}$ is a commitment to a length-0 tuple. For this, we only have to include to the CRS the elements $\stackrel{\circ}{g}_{1}$ and $\stackrel{\circ}{g}_{2}$. (The case $t=1$ can be handled dually.) The following theorem is basically a tautology, since the KE assumption states that the prover knows $r$. However, since any $\left(A_{2}, \AA_{2}\right)$, where $\stackrel{\circ}{A}_{2}=A_{2}^{\alpha}$, is a commitment of $\mathbf{0}$ (and thus, $\left.\left(\mathrm{gk} ; A_{2}\right) \in L\right)$ for some $r$, we cannot claim that Prot. 1 is computationally sound (even under a knowledge assumption). Instead, analogously to Gro10 Lip12, we prove a weaker version of soundness (which is however sufficient to achieve soundness of the shuffle argument). Note that the last statement of the theorem basically says that no efficient adversary can output an input to the product argument together with an accepting argument and openings to all commitments and all other pairs of type $(y, \bar{y})$ that are present in the argument, such that $a_{i} b_{i} \neq c_{i}$ for some $i$.

Theorem 1. The non-interactive zero argument in Prot. 1 is perfectly complete, perfectly zeroknowledge. Any non-uniform probabilistic-polynomial time adversary has a negligible chance of returning an input inp ${ }^{0}=A_{2}$ and a satisfying argument $\pi^{0}=\AA_{2}$ together with a opening witness $w^{0}=(\boldsymbol{a}, r)$, such that $\left(A_{2}, \AA_{2}\right)=\mathcal{C o m}^{2}\left(\mathrm{ck}_{2} ; \boldsymbol{a} ; r\right), \boldsymbol{a} \neq 0$ but the verification $\mathcal{V}_{0}\left(\mathrm{gk}, \mathrm{crs} ; A_{2}, \AA_{2}\right)$ accepts.

Proof. Perfect completeness is straightforward, since $\hat{e}\left(\stackrel{\circ}{g}_{1}, A_{2}\right)=\hat{e}\left(g_{1}^{\alpha}, A_{2}\right)=\hat{e}\left(g_{1}, A_{2}^{\alpha}\right)=\hat{e}\left(g_{1}, \AA_{2}\right)$. PERFECT ZERO-KNOWLEDGE: we construct the following simulator $\mathcal{S}=\left(\mathcal{S}_{1}, \mathcal{S}_{2}\right)$. The simulator $\mathcal{S}_{1}$ generates first $\mathrm{td}=\stackrel{\circ}{\alpha} \leftarrow \mathbb{Z}_{p}$, and then $\mathrm{crs} \leftarrow\left(\stackrel{\circ}{g}_{1} \leftarrow g_{1}^{\alpha}, \stackrel{\circ}{g}_{2} \leftarrow g_{2}^{\circ}\right)$, and saves $\mathrm{td}$. Since the simulator $\mathcal{S}_{2}$ later knows $\stackrel{\circ}{\alpha}$, it can compute a satisfying argument $\AA_{2}$ as $\AA_{2} \leftarrow A_{2}^{\alpha}$. Clearly, $\AA_{2}$ has the same distribution as in the real argument.

WEAKER VERSION OF SOUNDNESS: assume that there exists an adversary $\mathcal{A}$ that can break the last statement of the theorem. That is, $\mathcal{A}$ can create $\left(A_{2},(\boldsymbol{a}, r), \AA_{2}\right)$ such that $\left(A_{2}, \AA_{2}\right)=\mathcal{C} \operatorname{om}^{2}(\boldsymbol{a} ; r)$, $\boldsymbol{a} \neq \mathbf{0}$, and $\hat{e}\left(\stackrel{\circ}{g}_{1}, A_{2}\right)=\hat{e}\left(g_{1}, \stackrel{\circ}{A}_{2}\right)$. But then $\left(A_{2}, \stackrel{\circ}{A}_{2}\right)=\left(g_{2}^{r} \cdot \prod_{i=1}^{n} g_{2}^{a_{i} x^{\lambda_{i}}}, \stackrel{\circ}{g}_{2}^{r} \cdot \prod_{i=1}^{n} \stackrel{\circ}{g}_{2}^{a_{i} x^{\lambda_{i}}}\right)$ with $\lambda_{I} \neq 0$ for some $I \in[n]$. Since (gk, crs) contains $\stackrel{\circ}{g}_{2}^{x^{\ell}}$ only for $\ell \in\{0\}$, the adversary has thus broken the $\emptyset$-PSDL assumption. But the $\emptyset$-PSDL assumption is straightforwardly true, since then the input of the adversary does not depend on $x$ at all. Thus, the argument in Prot. 1 satisfies the last statement of the theorem.

The fact that the weaker version of soundness of this argument does not require any (non-trivial) assumption is, while somewhat surprising, also a logical consequence of CRS including $\stackrel{\circ}{g}_{2}^{x^{\ell}}$ only for $\ell \neq 0$. In fact, if the CRS contained $\stackrel{\circ}{g}_{2}^{x_{\ell}}$ for some other value of $\ell$ then the argument would not be sound under any (reasonable) computational assumption. The proof of the following lemma is straightforward.

Lemma 1. The CRS length in Prot. 1 is 1 element from the group $\mathbb{G}_{1}$ and 1 element from the group $\mathbb{G}_{2}$. The argument size in Prot. 1 is 1 element from the group $\mathbb{G}_{2}$. Prover's computational complexity is dominated by 1 exponentiation. The verifier's computational complexity is dominated by 2 bilinear pairings. 


\subsection{New 1-Sparsity Argument}

Assume that $A_{2} \in \mathbb{G}_{2}$. A vector $\boldsymbol{a} \in \mathbb{Z}_{p}^{n}$ is $k$-sparse, if it has at most $k$ non-zero coefficients. In a 1-sparsity argument in $\mathbb{G}_{2}$, the prover aims to convince the verifier that he knows an opening $A_{2}=g_{2}^{r} \cdot \prod_{i=1}^{n} g_{2, \lambda_{i}}^{a_{i}}$ such that $\boldsymbol{a}$ is 1-sparse, that is, there exists $I \in[n]$ such that for $i \neq I, a_{i}=0$, while $a_{I}$ can take any value, including 0 . Alternatively, since $\mathbb{Z}_{p}$ has no zero divisors, this means that the prover aims to convince the verifier that $a_{i} a_{j}=0$ for every $i, j \in[n]$ such that $i \neq j$. (Note that the zero argument can seen as a 0-sparsity argument.) A new 1-sparsity argument is depicted by Prot. 2 1-sparsity argument in $\mathbb{G}_{1}$ is defined dually.

Intuitively, the new 1-sparsity argument is constructed by following the same main ideas as the basic arguments (for Hadamard product and permutation) from [Lip12. That is, we start with a verification equation $\hat{e}\left(A_{1}, A_{2}\right)=\hat{e}\left(g_{1}, F\right)$, where the discrete logarithm of the left-hand side, see Eq. (1), is a sum of two polynomials $F_{\text {con }}(x)$ and $F_{\pi}(x)$, where $x$ is the secret key. In this case, $F_{c o n}(x)$ has $n(n-1)$ monomials (with coefficients $a_{i} a_{j}$ with $i \neq j$ ) that all vanish exactly if the prover is honest. On the other hand, the polynomial $F_{\pi}(x)$ has only $2 n+1$ monomials. Therefore, a honest prover can compute the argument given $2 n+1$ pairs $\left(g_{2 \ell}, \bar{g}_{2 \ell}\right)$. Moreover, the prover can construct $F$ by using 10 exponentiations. For comparison, in the basic arguments (the Hadamard product argument and the permutation argument) of Lip12, the polynomial $F_{\text {con }}(x)$ had $n$ monomials, and the polynomial $F_{\pi}(x)$ had $O\left(n 2^{2 \sqrt{2 \log _{2} n}}\right)=n^{1+o(1)}$ monomials. Thus, the CRS had $O\left(n 2^{2 \sqrt{2 \log _{2} n}}\right)=n^{1+o(1)}$ group elements and the prover's computational complexity was dominated by $O\left(n 2^{2 \sqrt{2 \log _{2} n}}\right)=n^{1+o(1)}$ exponentiations.

Similarly to the zero argument, we cannot prove the computational soundness of this argument, since for every $\boldsymbol{a}$, there exists $r$ such that $A_{2}=g_{2}^{r} \prod_{i \in[n]} g_{2}^{a_{i} x^{\lambda_{i}}}$. Instead, following Gro10 Lip12, we prove a weaker version of knowledge. Intuitively, the theorem statement includes $f_{\ell}^{\prime}$ only for $\ell \in \bar{\Lambda}$ (resp., $a_{\ell}$ for $\ell \in \Lambda$ together with $r$ ) since $\bar{g}_{2 \ell}$ (resp., $\bar{g}_{1 \ell}$ ) belongs to the CRS only for $\ell \in \bar{\Lambda}$ (resp., $\ell \in\{0\} \cup \Lambda$ ).

Theorem 2. The 1-sparsity argument in Prot. 2 is perfectly complete and perfectly witnessindistinguishable. Let $\Lambda$ be a progression-free set of odd positive integers. If the $\mathcal{G}_{\mathrm{bp}}$ is $\bar{\Lambda}$-PSDL secure, then any non-uniform PPT adversary has negligible chance of outputting inp ${ }^{\text {spa }} \leftarrow\left(A_{2}, \bar{A}_{2}\right)$ and a satisfying argument $\pi^{\text {spa }} \leftarrow\left(A_{1}, \bar{A}_{1}, F, \bar{F}\right)$ together with an opening witness $w^{\text {spa }} \leftarrow\left(\left(a_{\ell}\right)_{\ell \in \Lambda}, r,\left(f_{\ell}^{\prime}\right)_{\ell \in \bar{\Lambda}}\right)$, such that $\left(A_{2}, \bar{A}_{2}\right)=\mathcal{C} o m^{2}\left(\mathrm{ck}_{2} ; \boldsymbol{a} ; r\right),(F, \bar{F})=\left(g_{2}^{\sum_{\ell \in \bar{A}} f_{\ell}^{\prime} x_{\ell}}, \bar{g}_{2}^{\sum_{\ell \in \bar{\Lambda}} f_{\ell}^{\prime} x_{\ell}}\right)$, for some $i \neq j \in[n], a_{i} a_{j} \neq 0$, and the verification $\mathcal{V}_{\text {spa }}\left(\mathrm{gk}, \mathrm{crs} ;\left(A_{2}, \bar{A}_{2}\right), \pi^{\text {spa }}\right)$ accepts.

The (weak) soundness reduction is tight, except that it requires to factor a polynomial of degree $2 \lambda_{n}=$ $\max \{i \in \bar{\Lambda}\}$.

Proof. Let $\eta \leftarrow \hat{e}\left(A_{1}, A_{2}\right)$ and $h \leftarrow \hat{e}\left(g_{1}, g_{2}\right)$. PERFECT Witness-Indistinguishability: since satisfying argument $\pi^{s p a}$ is uniquely determined, all witnesses result in the same argument, and thus this argument is witness-indistinguishable.

PeRfeCt COMPLETEnEss. All verifications but the last one are straightforward. For the last verification $\hat{e}\left(A_{1}, A_{2}\right)=\hat{e}\left(g_{1}, F\right)$, note that $\log _{h} \eta=\left(r+\sum_{i=1}^{n} a_{i} x^{\lambda_{i}}\right)\left(r+\sum_{j=1}^{n} a_{j} x^{\lambda_{j}}\right)=F_{\text {con }}(x)+F_{\pi}(x)$, where

$$
F_{\text {con }}(x)=\underbrace{\sum_{i=1}^{n} \sum_{j=1: j \neq i}^{n} a_{i} a_{j} x^{\lambda_{i}+\lambda_{j}}}_{\delta \in 2 \Lambda} \text { and } F_{\pi}(x)=\underbrace{r^{2}+2 r \sum_{i=1}^{n} a_{i} x^{\lambda_{i}}+\sum_{i=1}^{n} a_{i}^{2} x^{2 \lambda_{i}}}_{\delta \in \bar{\Lambda}} .
$$

Thus, $\log _{h} \eta$ is equal to a sum of $x^{\delta}$ for $\delta \in 2 \Lambda$ and $\delta \in \bar{\Lambda}$. If the prover is honest, then $a_{i} a_{j}=0$ for $i \neq j$, and thus $\log _{h} \eta$ is a formal polynomial that has non-zero monomials $\gamma x^{\delta}$ with only $\delta \in \bar{\Lambda}$. Since then $a_{i}=0$ for $i \neq I$, we have $\log _{h} \eta=r^{2}+2 r a_{I} x^{\lambda_{I}}+a_{I}^{2} x^{2 \lambda_{I}}=\log _{g_{2}} F$. Thus, if the prover is honest, then the third verification succeeds.

WEAKER VERSION OF SOUNDNESS: Assume that $\mathcal{A}$ is an adversary that can break the last statement of the theorem. Next, we construct an adversary $\mathcal{A}^{\prime}$ against the $\bar{\Lambda}$-PSDL assumption. Let gk $\leftarrow \mathcal{G}_{\mathrm{bp}}\left(1^{\kappa}\right)$ and $x \leftarrow \mathbb{Z}_{p}$. The adversary $\mathcal{A}^{\prime}$ receives crs $\leftarrow\left(\mathrm{gk} ;\left(g_{1}^{x^{\ell}}, g_{2}^{x^{\ell}}\right)_{\ell \in \bar{\Lambda}}\right)$ as her input, and her task is to output $x$. She sets $\bar{\alpha} \leftarrow \mathbb{Z}_{p}$, crs $^{\prime} \leftarrow\left(\bar{g}_{1}, \bar{g}_{2},\left(g_{1}^{x^{\ell}}, g_{1}^{\bar{\alpha} x^{\ell}}\right)_{\ell \in \Lambda},\left(g_{2}^{x^{\ell}}, g_{2}^{\bar{\alpha} x^{\ell}}\right)_{\ell \in \Lambda \cup(2 \cdot \Lambda)}\right)$, and then forwards crs' to $\mathcal{A}$. Clearly, crs' follows the distribution imposed by $\mathcal{G}_{\text {crs }}\left(1^{\kappa}\right)$. Denote $\mathrm{ck}_{2} \leftarrow\left(\mathrm{gk} ; \bar{g}_{2},\left(g_{2}^{x^{\ell}}, g_{2}^{\bar{\alpha} x^{\ell}}\right)_{\ell \in \Lambda}\right)$. According 
System parameters: Let $n=\operatorname{poly}(\kappa)$. Let $\Lambda=\left\{\lambda_{i}: i \in[n]\right\}$ be an $(n, \kappa)$-nice progression-free set of odd positive integers. Denote $\lambda_{0}:=0$. Let $\bar{\Lambda}=\{0\} \cup \Lambda \cup(2 \cdot \Lambda)$.

CRS generation $\mathcal{G}_{\mathrm{crs}}\left(1^{\kappa}\right)$ : Let gk $:=\left(p, \mathbb{G}_{1}, \mathbb{G}_{2}, \mathbb{G}_{T}, \hat{e}, g_{1}, g_{2}\right) \leftarrow \mathcal{G}_{\mathrm{bp}}\left(1^{\kappa}\right)$. Let $\bar{\alpha}, x \leftarrow \mathbb{Z}_{p}$. Denote $\bar{g}_{t} \leftarrow g_{t}^{\bar{\alpha}}$, $g_{t \ell} \leftarrow g_{t}^{x^{\ell}}$ and $\bar{g}_{t \ell} \leftarrow g_{t}^{\bar{\alpha} x^{\ell}}$ for $t \in\{1,2\}$ and $\ell \in \bar{\Lambda}$. The CRS is crs $\leftarrow\left(\bar{g}_{1}, \bar{g}_{2},\left(g_{1 \ell}, \bar{g}_{1 \ell}\right)_{\ell \in \Lambda},\left(g_{2 \ell}, \bar{g}_{2 \ell}\right)_{\ell \in \Lambda \cup(2 \cdot \Lambda)}\right)$. Set $\mathrm{ck}_{2} \leftarrow\left(\mathrm{gk} ; \bar{g}_{2},\left(g_{2 \ell}, \bar{g}_{2 \ell}\right)_{\ell \in \Lambda}\right)$, and let $\mathrm{crs}_{v} \leftarrow\left(\bar{g}_{1}, \bar{g}_{2}\right)$ be the verifier's part of crs.

Common input: $\left(A_{2}, \bar{A}_{2}\right)=\mathcal{C o m}^{2}\left(\mathrm{ck}_{2} ; \boldsymbol{a} ; r\right)=\left(g_{2}^{r} \cdot g_{2, \lambda_{I}}^{a_{I}}, \bar{g}_{2}^{r} \cdot \bar{g}_{2, \lambda_{I}}^{a_{I}}\right) \in \mathbb{G}_{2}^{2}$, with $I \in[n]$.

Argument generation $\mathcal{P}_{\text {spa }}\left(\mathrm{gk}, \mathrm{crs} ;\left(A_{2}, \bar{A}_{2}\right),(\boldsymbol{a}, r)\right)$ : The prover defines $A_{1} \leftarrow g_{1}^{r} \cdot g_{1, \lambda_{I}}^{a_{I}}, \bar{A}_{1} \leftarrow \bar{g}_{1}^{r} \cdot \bar{g}_{1, \lambda_{I}}^{a_{I}}, F \leftarrow$ $g_{2}^{r^{2}} \cdot g_{2, \lambda_{I}}^{2 r a_{I}} \cdot g_{2,2 \lambda_{I}}^{a_{I}^{2}}$, and $\bar{F} \leftarrow \bar{g}_{2}^{r^{2}} \cdot \bar{g}_{2, \lambda_{I}}^{2 r a_{I}} \cdot \bar{g}_{2,2 \lambda_{I}}^{a_{I}^{2}}$. The prover sends $\pi^{s p a} \leftarrow\left(A_{1}, \bar{A}_{1}, F, \bar{F}\right) \in \mathbb{G}_{1}^{2} \times \mathbb{G}_{2}^{2}$ to the verifier as the argument.

Verification $\mathcal{V}_{\text {spa }}\left(\mathrm{gk}, \mathrm{crs}_{v} ;\left(A_{2}, \bar{A}_{2}\right), \pi^{\text {spa }}\right): \mathcal{V}_{\text {spa }}$ accepts iff $\hat{e}\left(A_{1}, g_{2}\right)=\hat{e}\left(g_{1}, A_{2}\right), \hat{e}\left(\bar{A}_{1}, g_{2}\right)=\hat{e}\left(A_{1}, \bar{g}_{2}\right)$, $\hat{e}\left(g_{1}, \bar{A}_{2}\right)=\hat{e}\left(\bar{g}_{1}, A_{2}\right), \hat{e}\left(g_{1}, \bar{F}\right)=\hat{e}\left(\bar{g}_{1}, F\right)$, and $\hat{e}\left(A_{1}, A_{2}\right)=\hat{e}\left(g_{1}, F\right)$.

Protocol 2: New 1-sparsity argument

to the last statement of the theorem, $\mathcal{A}\left(\mathrm{gk} ; \mathrm{crs}^{\prime}\right)$ returns $\left(\left(A_{2}, \bar{A}_{2}\right), w^{s p a}=\left(\left(a_{\ell}\right)_{\ell \in \Lambda}, r,\left(f_{\ell}^{\prime}\right)_{\ell \in \bar{\Lambda}}\right), \pi^{s p a}=\right.$ $\left.\left(A_{1}, \bar{A}_{1}, F, \bar{F}\right)\right)$.

Assume that $\mathcal{A}$ was successful, that is, for some $i, j \in[n]$ and $i \neq j, a_{i} a_{j} \neq 0$. Since $\left(A_{2}, \bar{A}_{2}\right)=$ $\mathcal{C o m}^{2}\left(\mathrm{ck}_{2} ; \boldsymbol{a} ; r\right)$ and $\mathcal{V}_{\text {spa }}\left(\mathrm{gk}, \mathrm{crs}^{\prime} ;\left(A_{2}, \bar{A}_{2}\right), \pi^{s p a}\right)=1, \mathcal{A}^{\prime}$ has expressed $\log _{h} \eta=\log _{g_{2}} F$ as a polynomial $f(x)$, where at least for some $\ell \in 2 \Upsilon \Lambda, x^{\ell}$ has a non-zero coefficient.

On the other hand, $\log _{g_{2}} F=\sum_{\ell \in \bar{\Lambda}} f_{\ell}^{\prime} x^{\ell}=f^{\prime}(x)$. Since $\Lambda$ is a progression-free set of odd positive integers, then $2 \wedge \cap \bar{\Lambda}=\emptyset$ and thus if $\ell \in \bar{\Lambda}$ then $\ell \notin 2 \Lambda$. Therefore, all coefficients of $f^{\prime}(x)$ corresponding to any $x^{\ell}, \ell \in 2 \wedge$, are equal to 0 . Thus $f(X)=\sum f_{\ell} X^{\ell}$ and $f^{\prime}(X)=\sum_{\ell \in \bar{\Lambda}} f_{\ell}^{\prime} X^{\ell}$ are different polynomials with

$$
f(x)=f^{\prime}(x)=\log _{g_{2}} F .
$$

Therefore, $\mathcal{A}^{\prime}$ has succeeded in creating a non-zero polynomial $d=f-f^{\prime}$, such that $d(x)=\sum_{\ell \in \bar{\Lambda}} d_{\ell} x^{\ell}=$ 0 .

Next, $\mathcal{A}^{\prime}$ can use an efficient polynomial factorization vHN10 algorithm in $\mathbb{Z}_{p}[X]$ to efficiently compute all $2 \lambda_{n}+1$ roots of $d(x)$. For some root $y, g_{1}^{x^{\ell}}=g_{1}^{y^{\ell}} . \mathcal{A}^{\prime}$ sets $x \leftarrow y$, thus violating the $\bar{\Lambda}$-PSDL assumption.

The 1-sparsity argument is not perfectly zero-knowledge. The problem is that the simulator knows $\mathrm{td}=(\bar{\alpha}, x)$, but given $\mathrm{td}$ and $\left(A_{2}, \bar{A}_{2}\right)$ she will not be able to generate $\pi^{s p a}$. E.g., she has to compute $A_{1}=g_{1}^{r} \cdot g_{1}^{a_{I} x^{\lambda_{I}}}$ based on $A_{2}=g_{2}^{r} \cdot g_{2}^{a_{I} x^{\lambda_{I}}}$ and $x$, but without knowing $r, I$ or $a_{I}$. This seems to be impossible without knowing an efficient isomorphism $\mathbb{G}_{1} \rightarrow \mathbb{G}_{2}$. Computing $F$ and $\bar{F}$ is even more difficult, since in this case the simulator does not even know the corresponding elements in $\mathbb{G}_{1}$. Technically, the problem is that due to the knowledge of the trapdoor, the simulator can, knowing one opening $(\boldsymbol{a}, r)$, produce an opening $\left(\boldsymbol{a}^{\prime}, r^{\prime}\right)$ to any other $\boldsymbol{a}^{\prime}$. However, here she does not know any openings. For the same reason, the permutation matrix argument of Sect. 3.3 will not be zero-knowledge. On the other hand, in the final shuffle argument of Sect. 5, the simulator creates all commitments by herself and can thus properly simulate the argument. By the same reason, the subarguments of [Gro10 Lip12] are not zero-knowledge but their final argument (for circuit satisfiability) is.

Theorem 3. Consider Prot. 2. The CRS consists of $2 n+1$ elements of $\mathbb{G}_{1}$ and $4 n+1$ elements of $\mathbb{G}_{2}$, with the verifier's part of the CRS consisting of only 1 element of $\mathbb{G}_{1}$ and 1 element of $\mathbb{G}_{2}$. The communication complexity (argument size) of the argument in Prot. 2 is 2 elements from $\mathbb{G}_{1}$ and 2 elements from $\mathbb{G}_{2}$. Prover's computational complexity is dominated by 10 exponentiations. Verifier's computational complexity is dominated by 10 bilinear pairings.

Proof. Straightforward. E.g., in a single 1-sparsity argument, the prover's (resp., the verifier's) computational complexity is dominated by 10 exponentiations (resp., pairings). In the case of the zero argument, the prover's (resp., the verifier's) computational complexity is dominated by 1 exponentiation (resp., 2 pairings).

\subsection{New Permutation Matrix Argument}

In this section, we will design a new permutation matrix argument where the prover aims to convince the verifier that he knows a permutation matrix $P$ such that $\left(c_{2 i}, \bar{c}_{2 i}\right) \in \mathbb{G}_{2}^{2}$ are knowledge commitments 
Setup: let gk := $\left(p, \mathbb{G}_{1}, \mathbb{G}_{2}, \mathbb{G}_{T}, \hat{e}, g_{1}, g_{2}\right) \leftarrow \mathcal{G}_{\text {bp }}\left(1^{\kappa}\right)$.

Common reference string $\mathcal{G}_{\text {crs }}(\mathrm{gk}):$ Let $\bar{\alpha}, \stackrel{\circ}{\alpha}, x \leftarrow \mathbb{Z}_{p}, \bar{g}_{t} \leftarrow g_{t}^{\bar{\alpha}}, \stackrel{\circ}{g}_{t} \leftarrow g_{t}^{\alpha}, g_{t \ell} \leftarrow g_{t}^{x^{\ell}}$, and $\bar{g}_{t \ell} \leftarrow \bar{g}_{t}^{x^{\ell}}$. Let $D \leftarrow \prod_{i=1}^{n} g_{2, \lambda_{i}}$. Let crs $\leftarrow\left(\bar{g}_{1}, \bar{g}_{2}, \stackrel{\circ}{1}_{1}, \stackrel{\circ}{2}_{2},\left(g_{1 \ell}, \bar{g}_{1 \ell}\right)_{\ell \in \Lambda},\left(g_{2 \ell}, \bar{g}_{2 \ell}\right)_{\ell \in \Lambda \cup(2 \cdot \Lambda)}, D\right)$, ck c $_{2}=\left(\mathrm{gk} ; \bar{g}_{2},\left(g_{2 \ell}, \bar{g}_{2 \ell}\right)_{\ell \in \Lambda}\right)$, $\mathrm{ck}_{2}=\left(\mathrm{gk} ; g_{2}, \stackrel{\circ}{g}_{2}\right)$, and $\mathrm{crs}_{v}=\left(\bar{g}_{1}, \bar{g}_{2}, \stackrel{\circ}{g}_{1}\right)$.

Common input: $\left(c_{2 i}, \bar{c}_{2 i}\right)=\mathcal{C o m}^{2}\left(\mathrm{ck}_{2} ; \boldsymbol{P}_{\boldsymbol{i}} ; r_{i}\right)=\left(g_{2}^{r_{i}} \cdot g_{2, \lambda_{\psi(i)}}, \bar{g}_{2}^{r_{i}} \cdot \bar{g}_{2, \lambda_{\psi(i)}}\right)$ for $i \in[n]$.

Argument Generation $\mathcal{P}_{p m}\left(\mathrm{gk}, \mathrm{crs} ;\left(\boldsymbol{c}_{\mathbf{2}}, \overline{\boldsymbol{c}}_{\mathbf{2}}\right),(P, \boldsymbol{r})\right)$ : Construct a zero argument $\pi^{0} \leftarrow \stackrel{\circ}{2}_{2}^{\sum_{i=1}^{n} r_{i}}$ that $\left(\prod_{i=1}^{n} c_{2 i}\right) / D$ commits to $\mathbf{0}$. For $i \in[n]$, construct a 1 -sparsity argument $\pi_{i}^{\text {spa }}=\left(c_{1 i}, \bar{c}_{1 i}, F_{i}, \bar{F}_{i}\right)$ that $\left(c_{2 i}, \bar{c}_{2 i}\right)$ commits to a 1 -sparse row. Send $\pi^{p m} \leftarrow\left(\pi^{0}, \boldsymbol{\pi}^{s p a}\right)$ to the verifier.

Verification $\mathcal{V}_{p m}\left(\mathrm{gk}, \mathrm{crs}_{v} ;\left(\boldsymbol{c}_{\mathbf{2}}, \overline{\boldsymbol{c}}_{\mathbf{2}}\right) ; \pi^{p m}\right)$ : The verifier checks $n+1 \operatorname{arguments}\left(\pi^{0}, \boldsymbol{\pi}^{\boldsymbol{s p a}}\right)$.

Protocol 3: New permutation matrix argument in group $\mathbb{G}_{2}$ with $P=P_{\psi}$

to $P$ 's rows. Recall that a permutation matrix is a Boolean matrix with exactly one 1 in every row and column: if $\psi$ is a permutation then the corresponding permutation matrix $P_{\psi}$ is such that $\left(P_{\psi}\right)_{i j}=1$ iff $j=\psi(i)$. Thus $\left(P_{\psi^{-1}}\right)_{i j}=1$ iff $i=\psi(j)$. We base our argument on the following lemma.

Lemma 2. An $n \times n$ matrix $P$ is a permutation matrix if and only if the following two conditions hold: (a) the sum of elements in any single column is equal to 1, and (b) no row has more than 1 non-zero elements.

Proof. First, assume that $P$ is a permutation matrix. Then every column has exactly one non-zero element (namely, with value 1), and thus both claims hold. Second, assume that (a) and (b) are true. Due to (a), every column must have at least one non-zero element, and thus the matrix has at least $n$ non-zero elements. Due to (b), no row has more than 1 non-zero elements, and thus the matrix has at most $n$ non-zero elements. Thus the matrix has exactly $n$ non-zero elements, one in each column. Due to (a), all non-zero elements are equal to 1 , and thus $P$ is a permutation matrix.

We now use the 1-sparsity argument and the zero argument to show that the committed matrix satisfies the claims of Lem. 2. Therefore, by Lem. 2, $P$ is a permutation matrix. Following Gro10 Lip12 and similarly to the case of the zero and 1-sparsity arguments, we prove that the permutation argument satisfies a "weaker" version of soundness.

Theorem 4. The argument in Prot. 3 is a perfectly complete and perfectly witness-indistinguishable permutation matrix argument. Let $\Lambda$ be a progression-free set of odd positive integers. If the $\bar{\Lambda}-P S D L$ assumption holds, then any non-uniform PPT adversary has a negligible chance in outputting an input inp ${ }^{\text {pm }} \leftarrow$ $\left(\boldsymbol{c}_{\mathbf{2}}, \overline{\boldsymbol{c}}_{\mathbf{2}}\right)$ and a satisfying argument $\pi^{p m} \leftarrow\left(\pi^{0},\left(c_{1 i}, \bar{c}_{1 i}, F_{i}, \bar{F}_{i}\right)_{i \in[n]}\right)$ together with an opening witness $w^{p m} \leftarrow\left(\left(a_{i}\right)_{i \in \Lambda}, r_{a},\left(\boldsymbol{P}_{i}, r_{i},\left(f_{i j}^{\prime}\right)_{j \in \bar{\Lambda}}\right)_{i \in[n]}\right)$, such that $\left(\left(\prod_{i=1}^{n} c_{2 i}\right) / D, \pi^{0}\right)=\mathcal{C o m}^{2}\left(\mathrm{ck}_{2} ; \boldsymbol{a} ; r_{a}\right),(\forall i \in$ $[n])\left(c_{2 i}, \bar{c}_{2 i}\right)=\mathcal{C o m}^{2}\left(\mathrm{ck}_{2} ; \boldsymbol{P}_{\boldsymbol{i}} ; r_{i}\right),(\forall i \in[n]) \log _{g_{2}} F_{i}=\sum_{j \in \bar{\Lambda}} f_{i j}^{\prime} x^{j},\left(\boldsymbol{a} \neq \mathbf{0} \vee(\exists i \in[n]) \boldsymbol{P}_{\boldsymbol{i}}\right.$ is not 1-sparse $)$, and the verification $\mathcal{V}_{p m}\left(\mathrm{gk}, \mathrm{crs} ;\left(\boldsymbol{c}_{\mathbf{2}}, \overline{\boldsymbol{c}}_{\mathbf{2}}\right), \pi^{p m}\right)$ accepts.

Proof. Perfect Completeness: follows from the completeness of the 1-sparsity and zero arguments and from Lem. 2, if we note that $\prod_{i=1}^{n} c_{2 i} / D=g_{2}^{\sum_{i=1}^{n} r_{i}}$, and thus $\left(\prod_{i=1}^{n} c_{2 i} / D, \pi^{0}\right)$ commits to 0 iff every column of $P$ sums to 1 .

WEAKER VERSION OF SOUNDNESS: Let $\mathcal{A}$ be a non-uniform PPT adversary that creates $\left(\boldsymbol{c}_{\mathbf{2}}, \overline{\boldsymbol{c}}_{\mathbf{2}}\right)$, an opening witness $\left(\left(a_{\ell}\right)_{\ell \in \Lambda}, r_{a},\left(P_{i}, r_{i},\left(f_{i j}^{\prime}\right)_{j \in \bar{\Lambda}}\right)_{i \in[n]}\right)$, and an accepting NIZK argument $\pi^{s p a}$.

Since the zero argument is (weakly) sound, verification of the argument $\pi^{0}$ shows that every column of $P$ sums to 1 . Here the witness is $w^{0}=\left(\boldsymbol{a}, r_{a}\right)$ with $\boldsymbol{a}=\sum_{i=1}^{n} \boldsymbol{P}_{\boldsymbol{i}}-\mathbf{1}$. By the $\bar{\Lambda}$-PSDL assumption, the 1 -sparsity assumption is (weakly) sound. Therefore, verification of the arguments $\boldsymbol{\pi}^{\boldsymbol{s} p \boldsymbol{a}}$ shows that every row of $P$ has exactly one 1 (here the witness is $\left.w_{i}^{s p a}=\left(P_{i}, r_{i},\left(f_{i j}^{\prime}\right)_{j \in \bar{\Lambda}}\right)\right)$. Therefore, by Lem. 2 and by the (weak) soundness of the 1-sparsity and zero arguments, $P$ is a permutation matrix.

PerfeCt Witness-INDistinguishability: since satisfying argument $\pi^{p m}$ is uniquely determined, all witnesses result in the same argument, and therefore the permutation matrix argument is witnessindistinguishable.

Lemma 3. Consider Prot. 3. The CRS consists of $2 n+2$ elements of $\mathbb{G}_{1}$ and $5 n+4$ elements of $\mathbb{G}_{2}$. The verifier's part of the CRS consists of 2 elements of $\mathbb{G}_{1}$ and of 2 elements of $\mathbb{G}_{2}$. The communication complexity is $2 n$ elements of $\mathbb{G}_{1}$ and $2 n+1$ elements of $\mathbb{G}_{2}$. The prover's computational complexity is 
dominated by $10 n+1$ exponentiations. The verifier's computational complexity is dominated by $10 n+2$ pairings.

Proof. Straightforward. E.g., in a single 1-sparsity argument, the prover's (resp., the verifier's) computational complexity is dominated by 10 exponentiations (resp., pairings). In the case of the zero argument, the prover's (resp., the verifier's) computational complexity is dominated by 1 exponentiation (resp., 2 pairings).

\section{Knowledge BBS Cryptosystem}

Boneh, Boyen and Shacham BBS04 proposed the BBS cryptosystem $\Pi=\left(\mathcal{G}_{\mathrm{bp}}, \mathcal{G}_{\mathrm{pkc}}, \mathcal{E}\right.$ nc, Dec). We will use a (publicly verifiable) "knowledge" version of this cryptosystem so that according to the KE (that is, the $\emptyset$-PKE) assumption, the party who produces a valid ciphertext must know both the plaintext and the randomizer. We give a definition for group $\mathbb{G}_{1}$, the knowledge BBS cryptosystem for group $\mathbb{G}_{2}$ can be defined dually.

Setup $\left(1^{\kappa}\right):$ Let gk $\leftarrow\left(p, \mathbb{G}_{1}, \mathbb{G}_{2}, \mathbb{G}_{T}, \hat{e}, g_{1}, g_{2}\right) \leftarrow \mathcal{G}_{\mathrm{bp}}\left(1^{\kappa}\right)$.

Key Generation $\mathcal{G}_{\text {pkc }}(\mathrm{gk}): \operatorname{Set}\left(\tilde{\alpha}_{1}, \tilde{\alpha}_{2}, \tilde{\alpha}_{3}\right) \leftarrow \mathbb{Z}_{p}^{3}, \tilde{g}_{1} \leftarrow g_{1}^{\tilde{\alpha}_{3}}, \tilde{g}_{2}^{(1)} \leftarrow g_{2}^{\tilde{\alpha}_{1}}, \tilde{g}_{2}^{(2)} \leftarrow g_{2}^{\tilde{\alpha}_{2}}, \tilde{g}_{2}^{(3)} \leftarrow g_{2}^{\tilde{\alpha}_{3}}$. The secret key is sk $:=\left(\mathrm{sk}_{1}, \mathrm{sk}_{2}\right) \leftarrow\left(\mathbb{Z}_{p}^{*}\right)^{2}$, and the public key is pk $\leftarrow\left(\mathrm{gk} ; \tilde{g}_{1}, \tilde{g}_{2}^{(1)}, \tilde{g}_{2}^{(2)}, \tilde{g}_{2}^{(3)}, f, \tilde{f}, h, \tilde{h}\right)$, where $f=g_{1}^{1 / \mathbf{s k}_{1}}, \tilde{f}=f^{\tilde{\alpha}_{1}}, h=g_{1}^{1 / \mathrm{sk}_{2}}$, and $\tilde{h}=h^{\tilde{\alpha}_{2}}$.

Encryption $\mathcal{E n c}_{\mathrm{pk}}(\mu ; \sigma, \tau)$ : To encrypt a message $\mu \in \mathbb{Z}_{p}$ with randomizer $(\sigma, \tau) \in \mathbb{Z}_{p}^{2}$, output the ciphertext $\mathfrak{u}=\left(\mathfrak{u}_{1}, \mathfrak{u}_{2}, \mathfrak{u}_{3}, \tilde{\mathfrak{u}}_{1}, \tilde{\mathfrak{u}}_{2}, \tilde{\mathfrak{u}}_{3}\right)$, where $\mathfrak{u}_{1}=f^{\sigma}, \mathfrak{u}_{2}=h^{\tau}, \mathfrak{u}_{3}=g_{1}^{\mu+\sigma+\tau}, \tilde{\mathfrak{u}}_{1}=\tilde{f}^{\sigma}$, and $\tilde{\mathfrak{u}}_{2}=\tilde{h}^{\tau}$, and $\tilde{\mathfrak{u}}_{3}=\tilde{g}_{1}^{\mu+\sigma+\tau}$.

Decryption $\mathcal{D e c} c_{\text {sk }}\left(\mathfrak{u}_{1}, \mathfrak{u}_{2}, \mathfrak{u}_{3}, \tilde{\mathfrak{u}}_{1}, \tilde{\mathfrak{u}}_{2}, \tilde{\mathfrak{u}}_{3}\right)$ : if $\hat{e}\left(\mathfrak{u}_{1}, \tilde{g}_{2}^{(1)}\right)=\hat{e}\left(\tilde{\mathfrak{u}}_{1}, g_{2}\right), \hat{e}\left(\mathfrak{u}_{2}, \tilde{g}_{2}^{(2)}\right)=\hat{e}\left(\tilde{\mathfrak{u}}_{2}, g_{2}\right)$ and $\hat{e}\left(\mathfrak{u}_{3}, \tilde{g}_{2}^{(3)}\right)=$ $\hat{e}\left(\tilde{\mathfrak{u}}_{3}, g_{2}\right)$, then return the discrete logarithm of $g_{1}^{\mu} \leftarrow \mathfrak{u}_{3} /\left(\mathfrak{u}_{1}^{\text {sk }} \mathfrak{u}_{2}^{\text {sk }}\right)$. Otherwise, return $\perp$.

Since $\mathcal{E} \mathrm{nc}_{\mathrm{pk}}\left(\mu_{1} ; \sigma_{1}, \tau_{1}\right) \cdot \mathcal{E} \mathrm{nc}_{\mathrm{pk}}\left(\mu_{2} ; \sigma_{2}, \tau_{2}\right)=\mathcal{E} \mathrm{nc}_{\mathrm{pk}}\left(\mu_{1}+\mu_{2} ; \sigma_{1}+\sigma_{2}, \tau_{1}+\tau_{2}\right)$, the knowledge BBS cryptosystem is additively homomorphic (with respect to element-wise multiplication of the ciphertexts). In particular, one can re-encrypt (that is, blind) a ciphertext efficiently: if $\sigma_{2}$ and $\tau_{2}$ are random, then $\mathcal{E n c}_{\mathrm{pk}}\left(\mu ; \sigma_{1}, \tau_{1}\right)$. $\mathcal{E n c}_{\mathrm{pk}}\left(0 ; \sigma_{2}, \tau_{2}\right)=\mathcal{E} \mathrm{nc}_{\mathrm{pk}}\left(\mu ; \sigma_{1}+\sigma_{2}, \tau_{1}+\tau_{2}\right)$ is a random encryption of $\mu$, independently of $\sigma_{1}$ and $\tau_{1}$.

The cryptosystem has to be lifted (i.e., the value $\mu$ be in exponent) for the soundness proof of the new shuffle argument in Sect. 5 to go through; see there for a discussion. Thus, to decrypt, one has to compute discrete logarithms. Since this the latter is intractable, in real applications one has to assume that $\mu$ is small. Consider for example the e-voting scenario where $\mu$ is the number of the candidate (usually a small number).

One can now use one of the following approaches. First, discard the ballots if the ciphertext does not decrypt. (This can be checked publicly.) Second, use a (non-interactive) range proof Bou00|LAN02 Lip03 CCs08|RKP09|CLs10|CLZ12] (in the e-voting scenario, range proofs are only given by the voters and not by the voting servers, and thus the range proof can be relatively less efficient compared to the shuffle argument) to guarantee that the ballots are correctly formed. In this case, invalid ballots can be removed from the system before starting to shuffle (saving thus valuable time otherwise wasted to shuffle invalid ciphertexts). Both approaches have their benefits, and either one can be used depending on the application.

The inclusion of $\tilde{\mathfrak{u}}_{3}$ to the ciphertext is required because of our proof technique. Without it, the extractor in the proof of of the soundness of the new shuffle argument can extract $\mu$ only if $\mu$ is small. Thus, security would not be guaranteed against an adversary who chooses $\mathfrak{u}_{3}$ without actually knowing the element $\mu$.

It is easy to see that the knowledge BBS cryptosystem, like the original BBS cryptosystem, is CPAsecure under the DLIN assumption (see Sect. A for the definition of the latter).

PRA1-Security. Under a knowledge (KE, that is, $\emptyset$-PKE) assumption the encrypting party knows the tuple $(\mu, \sigma, \tau)$ : there exists an extractor that returns $\sigma$ (resp., $\tau$ or $\mu$ ), given access to $\left(\mathfrak{u}_{1}, \tilde{\mathfrak{u}}_{1}\right)$ (resp., $\left(\mathfrak{u}_{2}, \tilde{\mathfrak{u}}_{2}\right)$ or $\left.\left(\mathfrak{u}_{3}, \tilde{\mathfrak{u}}_{3}\right)\right)$ and the encrypter's random coins. For this it is required that values $\tilde{\alpha}_{1}, \tilde{\alpha}_{2}$ and $\tilde{\alpha}_{3}$ are chosen independently at random. Thus, under the KE assumption in group $\mathbb{G}_{t}$, the knowledge-BBS cryptosystem satisfies PRA1-security, a version of plaintext-awareness (more precisely, PA1-security as defined in BP04]), where the extractor extracts both the plaintext and the randomizer. 


\section{New Shuffle Argument}

Let $\Pi=\left(\mathcal{G}_{\mathrm{pkc}}, \mathcal{E}\right.$ nc, $\mathcal{D}$ ec $)$ be an additively homomorphic cryptosystem. Assume that $\mathfrak{u}_{i}$ and $\mathfrak{u}_{i}^{\prime}$ are valid ciphertexts of $\Pi$. We say that $\left(\mathfrak{u}_{1}^{\prime}, \ldots, \mathfrak{u}_{n}^{\prime}\right)$ is a shuffle of $\left(\mathfrak{u}_{1}, \ldots, \mathfrak{u}_{n}\right)$ iff there exists a permutation $\psi \in S_{n}$ and randomizers $r_{1}, \ldots, r_{n}$ such that $\mathfrak{u}_{i}^{\prime}=\mathfrak{u}_{\psi(i)} \cdot \mathcal{E}$ nc $\mathrm{pk}\left(0 ; r_{i}\right)$ for $i \in[n]$. (In the case of the knowledge BBS cryptosystem, $r_{i}=\left(\sigma_{i}, \tau_{i}\right)$.) In a shuffle argument, the prover aims to convince the verifier in zeroknowledge that given $\left(\mathrm{pk},\left(\mathfrak{u}_{i}, \mathfrak{u}_{i}^{\prime}\right)_{i \in[n]}\right)$, he knows a permutation $\psi \in S_{n}$ and randomizers $r_{i}$ such that $\mathfrak{u}_{i}^{\prime}=\mathfrak{u}_{\psi(i)} \cdot \mathcal{E} \mathrm{nc}_{\mathrm{pk}}\left(0 ; r_{i}\right)$ for $i \in[n]$. More precisely, we define the group-specific binary relation $R^{s h}$ exactly as in GL07]:

$$
R^{s h}:=\left\{\left(\left(p, \mathbb{G}_{1}, \mathbb{G}_{2}, \mathbb{G}_{T}, \hat{e}, g_{1}, g_{2}\right),\left(\mathrm{pk},\left\{\mathfrak{u}_{i}\right\},\left\{\mathfrak{u}_{i}^{\prime}\right\}\right),\left(\psi,\left\{r_{i}\right\}\right)\right): \psi \in S_{n} \wedge\left(\forall i: \mathfrak{u}_{i}^{\prime}=\mathfrak{u}_{\psi(i)} \cdot \mathcal{E n c}_{\mathrm{pk}}\left(0 ; r_{i}\right)\right)\right\}
$$

Note that both according to the corresponding computational soundness definition and the Groth-Lu co-soundness definition (see App. B), the adversary picks not only the final ciphertexts $\mathfrak{u}_{i}^{\prime}$ but also the initial ciphertexts $\mathfrak{u}_{i}$.

In a real life application of the shuffle argument, the adversary (e.g., a malicious mix server) usually gets the ciphertexts $\mathfrak{u}_{i}$ from a third party (from voters, or from another mix server), and thus does not know their discrete logarithms. However, in such a case we can still prove soundness of the full e-voting system (including the voters and all mix servers) if we give the adversary access to secret coins of all relevant parties. The use of knowledge BBS guarantees that the encrypters (voters) know the plaintexts and the randomizers, and thus the use of knowledge BBS can be seen as a white-box non-interactive knowledge argument. This corresponds to the case in several interactive (or Fiat-Shamir heuristic based) shuffles, where the ballots are accompanied by a proof of knowledge of the actual vote, from what the (black-box) simulator obtains the actual plaintexts necessary to complete the simulation. We thus think that soundness in our model is relevant, and corresponds to the established cryptographic practice with a twist. We leave the question of whether this model is necessary in applications like e-voting (where initial ciphertexts are not provided by the mixservers), and when co-soundness is undesired, as an interesting open problem. Using the Groth-Lu co-soundness definition avoids this issue, since in that case the adversary does not have access to the random coins of the participants.

We note that Groth and Lu made in addition a similar assumption in GL07 where they prove cosoundness against adversaries who also output and thus know the secret key of the cryptosystem. (See App. B for a precise definition.) Thus, the adversary can decrypt all the ciphertexts, and thus knows the plaintexts (but does not have to know the randomizers). As argued in GL07, this is reasonable in the setting of mixnet where the servers can usually threshold-decrypt all the results. Their approach is however not applicable in our case, since the knowledge of the secret key enables the adversary to obtain the plaintexts and the randomizers in exponents, while to prove the soundness in Thm. 5 the adversary has to know the plaintexts and the randomizers themselves.

Next, we construct an efficient shuffle argument that works with the knowledge BBS cryptosystem of Sect. 4. Assume that the ciphertexts $\left(\mathfrak{u}_{i 1}, \mathfrak{u}_{i 2}, \mathfrak{u}_{i 3}, \tilde{\mathfrak{u}}_{i 1}, \tilde{\mathfrak{u}}_{i 2}, \tilde{\mathfrak{u}}_{i 3}\right)$, where $i \in[n]$, are created as in Sect. 4 . The shuffled ciphertexts with permutation $\psi \in S_{n}$ and randomizers $\left(\sigma_{i}^{\prime}, \tau_{i}^{\prime}\right)_{i \in[n]}$ are

$$
\mathfrak{u}_{i}^{\prime}=\left(\mathfrak{u}_{i 1}^{\prime}, \mathfrak{u}_{i 2}^{\prime}, \mathfrak{u}_{i 3}^{\prime}, \tilde{\mathfrak{u}}_{i 1}^{\prime}, \tilde{\mathfrak{u}}_{i 2}^{\prime}, \tilde{\mathfrak{u}}_{i 3}^{\prime}\right)=\mathfrak{u}_{\psi(i)} \cdot \mathcal{E} \mathrm{nc}_{\mathrm{pk}}\left(0 ; \sigma_{i}^{\prime}, \tau_{i}^{\prime}\right)=\mathcal{E} \mathrm{nc}_{\mathrm{pk}}\left(\mu_{\psi(i)} ; \sigma_{\psi(i)}+\sigma_{i}^{\prime}, \tau_{\psi(i)}+\tau_{i}^{\prime}\right) .
$$

Let $P=P_{\psi^{-1}}$ denote the permutation matrix corresponding to the permutation $\psi^{-1}$.

The new shuffle argument is described in Prot. 4. Here, the prover first constructs a permutation matrix and a permutation matrix argument $\pi^{p m}$. After that, he shows that the plaintext vector of $\mathfrak{u}_{i}^{\prime}$ is equal to the product of this permutation matrix and the plaintext vector of $\mathfrak{u}_{i}$. Importantly, we can prove the adaptive computational soundness of the shuffle argument. This is since while in the previous arguments one only relied on (perfectly hiding) knowledge commitment scheme and thus any commitment could commit at the same time to the correct value (for example, to a permutation matrix) and to an incorrect value (for example, to an all-zero matrix), here the group-dependent language contains statements about a public-key cryptosystem where any ciphertext can be uniquely decrypted. Thus, it makes sense to state that $\left(\mathrm{pk},\left(\mathfrak{u}_{i}, \mathfrak{u}_{i}^{\prime}\right)_{i \in[n]}\right)$ is not a shuffle. To prove computational soundness, we need to rely on the PKE assumption. It is also nice to have a shuffle argument that satisfies a standard security notion.

Theorem 5. Prot. 4 is a non-interactive perfectly complete and perfectly zero-knowledge shuffle argument of the knowledge BBS ciphertexts. Assume that $\mu$ is sufficiently small so that $\log _{g_{1}} g_{1}^{\mu}$ can be 
Common reference string: Similarly to the permutation matrix argument, let $\bar{\alpha}, \stackrel{\circ}{\alpha}, x \leftarrow \mathbb{Z}_{p}, \bar{g}_{t} \leftarrow g_{t}^{\bar{\alpha}}, \stackrel{\circ}{g}_{t} \leftarrow g_{t}^{\alpha}$, $g_{t \ell} \leftarrow g_{t}^{x^{\ell}}$, and $\bar{g}_{t \ell} \leftarrow \bar{g}_{t}^{x^{\ell}}$. Let $D \leftarrow \prod_{i=1}^{n} g_{2, \lambda_{i}}$. In addition, let $\mathrm{sk}_{1}, \mathrm{sk}_{2} \leftarrow \mathbb{Z}_{p}^{*}$ and $\tilde{\alpha}_{1}, \tilde{\alpha}_{2}, \tilde{\alpha}_{3} \leftarrow \mathbb{Z}_{p}$. Let $f \leftarrow g_{1}^{1 / \mathrm{sk}_{1}}, h \leftarrow g_{1}^{1 / \mathrm{sk}_{2}}, \tilde{f} \leftarrow f^{\tilde{\alpha}_{1}}, \tilde{h} \leftarrow h^{\tilde{\alpha}_{2}}, \tilde{g}_{1} \leftarrow g_{1}^{\tilde{\alpha}_{3}}, \tilde{g}_{2}^{(1)} \leftarrow g_{2}^{\tilde{\alpha}_{1}}, \tilde{g}_{2}^{(2)} \leftarrow g_{2}^{\tilde{\alpha}_{2}}$, and $\tilde{g}_{2}^{(3)} \leftarrow g_{2}^{\tilde{\alpha}_{3}}$.

The CRS is

$$
\text { crs : }=\left(\bar{g}_{1}, \bar{g}_{2}, \stackrel{\circ}{g}_{1}, \stackrel{\circ}{g}_{2},\left(g_{1 \ell}, \bar{g}_{1 \ell}\right)_{\ell \in \Lambda},\left(g_{2 \ell}, \bar{g}_{2 \ell}\right)_{\ell \in \Lambda \cup(2 \cdot \Lambda)}, D\right) \text {. }
$$

The commitment keys are $\mathrm{ck}_{t} \leftarrow\left(\mathrm{gk} ; \bar{g}_{t},\left(g_{t \ell}, \bar{g}_{t \ell}\right)_{\ell \in \Lambda}\right)$ and $\mathrm{ck}_{2} \leftarrow\left(\mathrm{gk} ; \stackrel{\circ}{g}_{2}\right)$. The public key is pk= $\left(\mathrm{gk} ; \tilde{g}_{1}, \tilde{g}_{2}^{(1)}, \tilde{g}_{2}^{(2)}, \tilde{g}_{2}^{(3)}, f, \tilde{f}, h, \tilde{h}\right)$, and the secret key is sk $=\left(\mathrm{sk}_{1}, \mathrm{sk}_{2}\right)$.

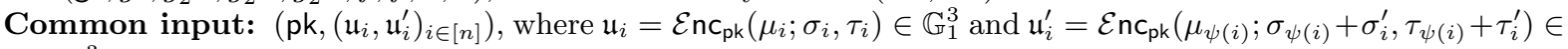
$\mathbb{G}_{1}^{3}$.

Argument $\mathcal{P}_{s h}\left(\mathrm{gk}, \mathrm{crs} ;\left(\mathrm{pk},\left(\mathfrak{u}_{i}, \mathfrak{u}_{i}^{\prime}\right)_{i \in[n]}\right),\left(\psi,\left(\sigma_{i}^{\prime}, \tau_{i}^{\prime}\right)_{i \in[n]}\right)\right)$ : the prover does the following.

1. Let $P=P_{\psi^{-1}}$ be the $n \times n$ permutation matrix corresponding to the permutation $\psi^{-1}$.

2. For $i \in[n]$, let $r_{i} \leftarrow \mathbb{Z}_{p}$ and $\left(c_{2 i}, \bar{c}_{2 i}\right) \leftarrow \mathcal{C o m}^{2}\left(\mathrm{ck}_{2} ; \boldsymbol{P}_{\boldsymbol{i}} ; r_{i}\right)=\left(g_{2}^{r_{i}} \cdot g_{2, \lambda_{\psi^{-1}(i)}}, \bar{g}_{2}^{r_{i}} \cdot \bar{g}_{2, \lambda_{\psi^{-1}(i)}}\right)$.

3. Generate a permutation matrix argument $\pi^{p m}$ for inputs $\left(\boldsymbol{c}_{\mathbf{2}}, \overline{\boldsymbol{c}}_{\mathbf{2}}\right)$.

4. Set $\left(R_{\sigma}, R_{\tau}\right) \leftarrow \mathbb{Z}_{p}^{2},\left(c_{\sigma}, \bar{c}_{\sigma}\right) \leftarrow \mathcal{C o m}^{2}\left(\mathrm{ck}_{2} ; \sigma_{1}^{\prime}, \ldots, \sigma_{n}^{\prime} ; R_{\sigma}\right)$, and $\left(c_{\tau}, \bar{c}_{\tau}\right) \leftarrow \mathcal{C o m}^{2}\left(\mathrm{ck}_{2} ; \tau_{1}^{\prime}, \ldots, \tau_{n}^{\prime} ; R_{\tau}\right)$.

5. Compute $\left(\mathfrak{u}_{\sigma}, \tilde{\mathfrak{u}}_{\sigma}\right) \leftarrow\left(f^{R_{\sigma}} \cdot \prod_{i=1}^{n} \mathfrak{u}_{i 1}^{r_{i}}, \tilde{f}^{R_{\sigma}} \cdot \prod_{i=1}^{n} \tilde{\mathfrak{u}}_{i 1}^{r_{i}}\right),\left(\mathfrak{u}_{\tau}, \tilde{\mathfrak{u}}_{\tau}\right) \leftarrow\left(h^{R_{\tau}} \cdot \prod_{i=1}^{n} \mathfrak{u}_{i 2}^{r_{i}}, \tilde{h}^{R_{\tau}} \cdot \prod_{i=1}^{n} \tilde{\mathfrak{u}}_{i 2}^{r_{i}}\right),\left(\mathfrak{u}_{\mu}, \tilde{\mathfrak{u}}_{\mu}\right) \leftarrow$ $\left(g_{1}^{R_{\sigma}+R_{\tau}} \cdot \prod_{i=1}^{n} \mathfrak{u}_{i 3}^{r_{i}}, \tilde{g}_{1}^{R_{\sigma}+R_{\tau}} \cdot \prod_{i=1}^{n} \tilde{\mathfrak{u}}_{i 3}^{r_{i}}\right)$.

6. The argument is

$$
\pi^{s h} \leftarrow\left(\left(c_{2 i}, \bar{c}_{2 i}\right)_{i \in[n]}, \pi^{p m}, c_{\sigma}, \bar{c}_{\sigma}, c_{\tau}, \bar{c}_{\tau}, \mathfrak{u}_{\sigma}, \tilde{\mathfrak{u}}_{\sigma}, \mathfrak{u}_{\tau}, \tilde{\mathfrak{u}}_{\tau}, \mathfrak{u}_{\mu}, \tilde{\mathfrak{u}}_{\mu}\right) .
$$

Verification $\mathcal{V}_{s h}\left(\mathrm{gk}, \mathrm{crs} ;\left(\mathrm{pk},\left(\mathfrak{u}_{i}, \mathfrak{u}_{i}^{\prime}\right)_{i \in[n]}\right), \pi^{s h}\right)$ : the verifier does the following.

1. Check that $\hat{e}\left(\bar{g}_{1}, c_{\sigma}\right)=\hat{e}\left(g_{1}, \bar{c}_{\sigma}\right)$ and $\hat{e}\left(\bar{g}_{1}, c_{\tau}\right)=\hat{e}\left(g_{1}, \bar{c}_{\tau}\right)$. // $\left(c_{\sigma}, \bar{c}_{\sigma}\right)$ and $\left(c_{\tau}, \bar{c}_{\tau}\right)$ are correct.

2. Check that $\hat{e}\left(\mathfrak{u}_{\sigma}, \tilde{g}_{2}^{(1)}\right)=\hat{e}\left(\tilde{\mathfrak{u}}_{\sigma}, g_{2}\right), \hat{e}\left(\mathfrak{u}_{\tau}, \tilde{g}_{2}^{(2)}\right)=\hat{e}\left(\tilde{\mathfrak{u}}_{\tau}, g_{2}\right)$, and $\hat{e}\left(\mathfrak{u}_{\mu}, \tilde{g}_{2}^{(3)}\right)=\hat{e}\left(\tilde{\mathfrak{u}}_{\mu}, g_{2}\right)$.

3. For $i \in[n]$, check that $\hat{e}\left(\mathfrak{u}_{i 1}, \tilde{g}_{2}^{(1)}\right)=\hat{e}\left(\tilde{\mathfrak{u}}_{i 1}, g_{2}\right), \hat{e}\left(\mathfrak{u}_{i 2}, \tilde{g}_{2}^{(2)}\right)=\hat{e}\left(\tilde{\mathfrak{u}}_{i 2}, g_{2}\right), \hat{e}\left(\mathfrak{u}_{i 3}, \tilde{g}_{2}^{(3)}\right)=\hat{e}\left(\tilde{\mathfrak{u}}_{i 3}, g_{2}\right)$, $\hat{e}\left(\mathfrak{u}_{i 1}^{\prime}, \tilde{g}_{2}^{(1)}\right)=\hat{e}\left(\tilde{\mathfrak{u}}_{i 1}^{\prime}, g_{2}\right), \hat{e}\left(\mathfrak{u}_{i 2}^{\prime}, \tilde{g}_{2}^{(2)}\right)=\hat{e}\left(\tilde{\mathfrak{u}}_{i 2}^{\prime}, g_{2}\right)$, and $\hat{e}\left(\mathfrak{u}_{i 3}^{\prime}, \tilde{g}_{2}^{(3)}\right)=\hat{e}\left(\tilde{\mathfrak{u}}_{i 3}^{\prime}, g_{2}\right)$. // Ciphertexts are correct.

4. Check the permutation matrix argument $\pi^{p m}$.

5. Check that the following three equations hold:
(a) $\hat{e}\left(f, c_{\sigma}\right) \cdot \prod_{i=1}^{n} \hat{e}\left(\mathfrak{u}_{i 1}, c_{2 i}\right)=\hat{e}\left(\mathfrak{u}_{\sigma}, g_{2}\right) \cdot \prod_{i=1}^{n} \hat{e}\left(\mathfrak{u}_{i 1}^{\prime}, g_{2, \lambda_{i}}\right)$,
(b) $\hat{e}\left(h, c_{\tau}\right) \cdot \prod_{i=1}^{n} \hat{e}\left(\mathfrak{u}_{i 2}, c_{2 i}\right)=\hat{e}\left(\mathfrak{u}_{\tau}, g_{2}\right) \cdot \prod_{i=1}^{n} \hat{e}\left(\mathfrak{u}_{i 2}^{\prime}, g_{2, \lambda_{i}}\right)$, and
(c) $\hat{e}\left(g_{1}, c_{\sigma} c_{\tau}\right) \cdot \prod_{i=1}^{n} \hat{e}\left(\mathfrak{u}_{i 3}, c_{2 i}\right)=\hat{e}\left(\mathfrak{u}_{\mu}, g_{2}\right) \cdot \prod_{i=1}^{n} \hat{e}\left(\mathfrak{u}_{i 3}^{\prime}, g_{2, \lambda_{i}}\right)$.

Protocol 4: New shuffle argument

computed in polynomial time. If the $\Lambda-P S D L$, the DLIN, the $K E$ (in group $\mathbb{G}_{1}$ ), and the $\bar{\Lambda}-P K E$ (in group $\mathbb{G}_{2}$ ) assumptions hold, then the argument is also adaptively computationally sound.

We recall that $\emptyset$-PKE is equal to the $\mathrm{KE}$ assumption (in the same bilinear group). Thus, if $\bar{\Lambda}$-PKE is hard then also $\Lambda$-PKE and KE are hard (in the same group).

Proof. Perfect COmpleteness: To verify the proof, the verifier first checks the consistency of the commitments, ciphertexts and the permutation matrix argument; here one needs that the permutation matrix argument is perfectly complete. Assume that the prover is honest. The verification equation in step $5 \mathrm{a}$ holds since

$$
\begin{aligned}
\hat{e}\left(f, c_{\sigma}\right) \cdot \prod_{i=1}^{n} \hat{e}\left(\mathfrak{u}_{i 1}, c_{2 i}\right) & =\hat{e}\left(f, g_{2}^{R_{\sigma}} \cdot \prod_{i=1}^{n} g_{2, \lambda_{i}}^{\sigma_{i}^{\prime}}\right) \cdot \prod_{i=1}^{n}\left(\hat{e}\left(\mathfrak{u}_{i 1}, g_{2}^{r_{i}}\right) \cdot \hat{e}\left(f^{\sigma_{i}}, g_{2, \lambda_{\psi}-1(i)}\right)\right) \\
& =\hat{e}\left(f^{R_{\sigma}} \cdot \prod_{i=1}^{n} \mathfrak{u}_{i 1}^{r_{i}}, g_{2}\right) \cdot \prod_{i=1}^{n} \hat{e}\left(f^{\sigma_{\psi(i)}+\sigma_{i}^{\prime}}, g_{2, \lambda_{i}}\right) \\
& =\hat{e}\left(\mathfrak{u}_{\sigma}, g_{2}\right) \cdot \prod_{i=1}^{n} \hat{e}\left(\mathfrak{u}_{i 1}^{\prime}, g_{2, \lambda_{i}}\right) .
\end{aligned}
$$

The equations in steps $5 \mathrm{~b}$ and $5 \mathrm{c}$ can be verified similarly.

AdAPtive COMPUTATIONAL SOUNDNEss: Let $\mathcal{A}$ be a non-uniform PPT adversary that, given gk and a crs, creates a statement $\left(\mathrm{pk}=\left(\mathrm{gk} ; \tilde{g}_{1}, \tilde{g}_{2}^{(1)}, \tilde{g}_{2}^{(2)}, \tilde{g}_{2}^{(3)}, f, \tilde{f}, h, \tilde{h}\right),\left(\mathfrak{u}_{i}, \mathfrak{u}_{i}^{\prime}\right)_{i \in[n]}\right)$ and an accepting NIZK argument $\pi^{s h}$ (as in Eq. (2) in Prot. 4), such that the plaintext vector $\left(\mathfrak{u}_{i}^{\prime}\right)_{i \in[n]}$ is not a permutation of the plaintext vector $\left(\mathfrak{u}_{i}\right)_{i \in[n]}$. Assume that the DLIN assumption holds in $\mathbb{G}_{1}$, the KE assumption 
holds in $\mathbb{G}_{1}$ and $\bar{\Lambda}$-PKE (and thus also $\Lambda$-PKE and KE) assumption holds in $\mathbb{G}_{2}$. We now construct an adversary $\mathcal{A}^{\prime}$ that breaks the $\Lambda$-PSDL assumption.

Recall that $\pi^{p m}$ contains values $\pi^{0}$ and $\pi_{i}^{s p a}=\left(c_{1 i}, \bar{c}_{1 i}, F_{i}, \bar{F}_{i}\right)$. By applying the relevant knowledge assumption, we can postulate the existence of the following non-uniform PPT knowledge extractors that, with all but a negligible probability, return certain values:

- By the $\mathrm{KE}$ assumption in group $\mathbb{G}_{1}$, there exists a knowledge extractor that, given $\left(\mathfrak{u}_{i j}, \tilde{\mathfrak{u}}_{i j}, \mathfrak{u}_{i j}^{\prime}, \tilde{\mathfrak{u}}_{i j}^{\prime}\right)_{j \in[3]}$ and access to $\mathcal{A}$ 's random coins, returns the values $\mu_{i}, \sigma_{i}, \tau_{i}, \mu_{i}^{\prime}, \sigma_{i}^{\prime}$ and $\tau_{i}^{\prime}$, such that $\mathfrak{u}_{i}=\mathcal{E} \mathrm{nc}_{\mathrm{pk}}\left(\mu_{i} ; \sigma_{i}, \tau_{i}\right)$ and $\mathfrak{u}_{i}^{\prime}=\mathcal{E} \mathrm{nc}_{\mathrm{pk}}\left(\mu_{i}^{\prime} ; \sigma_{i}^{\prime}, \tau_{i}^{\prime}\right)$. Note that it might be the case that $\mu_{i}^{\prime} \neq \mu_{\varrho(i)}$.

- By the $\Lambda$-PKE assumption in group $\mathbb{G}_{2}$, there exists a knowledge extractor that, given $\left(c_{\sigma}, \bar{c}_{\sigma}, c_{\tau}, \bar{c}_{\tau}\right)$ and access to $\mathcal{A}$ 's random coins, returns openings $\left(\sigma^{*}, R_{\sigma}\right)$ and $\left(\boldsymbol{\tau}^{*}, R_{\tau}\right)$, such that $\left(c_{\sigma}, \bar{c}_{\sigma}\right)=$ $\mathcal{C o m}^{2}\left(\mathrm{ck}_{2} ; \boldsymbol{\sigma}^{*} ; R_{\sigma}\right)$ and $\left(c_{\tau}, \bar{c}_{\tau}\right)=\mathcal{C o m}^{2}\left(\mathrm{ck}_{2} ; \tau^{*} ; R_{\tau}\right)$. It does not have to hold that $\sigma_{i}^{\prime}=\sigma_{\psi(i)}+\sigma_{i}^{*}$ and $\tau_{i}^{\prime}=\tau_{\psi(i)}+\tau_{i}^{*}$ for $i \in[n]$.

- By the $\mathrm{KE}$ assumption in group $\mathbb{G}_{1}$, there exists a knowledge extractor that, given $\left(\mathfrak{u}_{\sigma}, \tilde{\mathfrak{u}}_{\sigma}, \mathfrak{u}_{\tau}, \tilde{\mathfrak{u}}_{\tau}, \mathfrak{u}_{\mu}, \tilde{\mathfrak{u}}_{\mu}\right)$ and access to $\mathcal{A}$ 's random coins, returns openings $\left(v_{\sigma}, v_{\tau}, v_{\mu}\right)$, such that $\left(\mathfrak{u}_{\sigma}, \tilde{\mathfrak{u}}_{\sigma}\right)=\left(f^{v_{\sigma}}, \tilde{f}^{v_{\sigma}}\right),\left(\mathfrak{u}_{\tau}, \tilde{\mathfrak{u}}_{\tau}\right)=\left(h^{v_{\tau}}, \tilde{h}^{v_{\tau}}\right)$, and $\left(\mathfrak{u}_{\mu}, \tilde{\mathfrak{u}}_{\mu}\right)=\left(g_{1}^{v_{\mu}}, \tilde{g}_{1}^{v_{\mu}}\right)$. (Thus, it is not necessary that the adversary created the values $\mathfrak{u}_{\sigma}, \mathfrak{u}_{\tau}$ and $\mathfrak{u}_{\mu}$ correctly, it is just needed that she knows their discrete logarithms.)

- By the KE assumption in group $\mathbb{G}_{2}$, there exists a knowledge extractor that, given $\left(\left(\prod_{i=1}^{n} c_{2 i}\right) / D, \pi^{0}\right)$ and access to $\mathcal{A}$ 's random coins, returns an opening $\left(\left(a_{i}\right)_{i \in[n]}, r_{a}\right)$, such that $\left(\left(\prod_{i=1}^{n} c_{2 i}\right) / D, \pi^{0}\right)=$ $\mathrm{Com}^{2}\left(\mathrm{ck}_{2} ; \boldsymbol{a} ; r_{a}\right)$.

- By the $\Lambda$-PKE assumption in group $\mathbb{G}_{2}$, for every $i \in[n]$ there exists a knowledge extractor that, given $\left(c_{2 i}, \bar{c}_{21}\right)$ and access to $\mathcal{A}$ 's random coins, returns an opening $\left(\left(P_{i j}\right)_{j \in[n]}, r_{i}\right)$ such that $\left(c_{2 i}, \bar{c}_{2 i}\right)=$ $\mathrm{Com}^{2}\left(\mathrm{ck}_{2} ; \boldsymbol{P}_{\boldsymbol{i}} ; r_{i}\right)$.

- By the $\bar{\Lambda}$-PKE assumption in group $\mathbb{G}_{2}$, for every $i$ there exists a knowledge extractor that, given $\left(F_{i}, \bar{F}_{i}\right)$ and access to $\mathcal{A}$ 's random coins, returns openings $\left(f_{i j}^{\prime}\right)_{j \in \bar{\Lambda}}$ such that $\log _{g_{2}} F_{i}=\sum_{j \in \bar{\Lambda}} f_{i j}^{\prime} x^{j}$.

The probability that any of these extractors fails is negligible, in this case we can abort. In the following, we will assume that all extractors succeeded.

Let $\mathfrak{a}$ be $\mathcal{A}$ 's output. Based on $\mathcal{A}$ and the last three type of extractors, we can build an adversary $\mathcal{A}^{\prime}$ that returns $\mathfrak{a}$ together with $\left(\left(a_{i}\right)_{i \in[n]}, r_{a},\left(\boldsymbol{P}_{\boldsymbol{i}}, r_{i},\left(f_{i j}^{\prime}\right)_{j \in \bar{\Lambda}}\right)_{i \in[n]}\right)$. Since the permutation matrix argument is (weakly) sound (as defined in the last statement of Thm. (4) and $\pi^{p m}$ verifies, we have that $\boldsymbol{c}_{\mathbf{2}}=$ $\left(c_{2 i}\right)_{i \in[n]}$ commits to a permutation matrix. Thus, there exists $\psi \in S_{n}$ such that for every $i \in[n]$, $c_{2 i}=\exp \left(g_{2}, r_{i}+x^{\left.\lambda_{\left(\psi^{-1}(i)\right)}\right)}\right.$.

Assume now that the equation in step 5 a holds. Then

$$
\begin{aligned}
\hat{e}\left(\mathfrak{u}_{\sigma}, g_{2}\right) & =\hat{e}\left(f, c_{\sigma}\right) \cdot \prod_{i=1}^{n} \hat{e}\left(\mathfrak{u}_{i 1}, c_{2 i}\right) / \prod_{i=1}^{n} \hat{e}\left(\mathfrak{u}_{i 1}^{\prime}, g_{2, \lambda_{i}}\right) \\
& =\hat{e}\left(f, g_{2}^{R_{\sigma}+\sum_{i=1}^{n} \sigma_{i}^{*} x^{\lambda_{i}}}\right) \cdot \prod_{i=1}^{n} \hat{e}\left(f^{\sigma_{i}}, g_{2}^{r_{i}+x^{\lambda} \psi^{-1}(i)}\right) / \prod_{i=1}^{n} \hat{e}\left(f^{\sigma_{i}^{\prime}}, g_{2}^{x^{\lambda_{i}}}\right) \\
& =\hat{e}\left(f^{R_{\sigma}+\sum_{i=1}^{n} \sigma_{i} r_{i}+\sum_{i=1}^{n}\left(\sigma_{\psi(i)}+\sigma_{i}^{*}-\sigma_{i}^{\prime}\right) x^{\lambda_{i}}}, g_{2}\right) .
\end{aligned}
$$

Since $\mathfrak{u}_{\sigma}=f^{v_{\sigma}}, \sum_{i=1}^{n}\left(\sigma_{\psi(i)}+\sigma_{i}^{*}-\sigma_{i}^{\prime}\right) x^{\lambda_{i}}+R_{\sigma}+\sum_{i=1}^{n} \sigma_{i} r_{i}-v_{\sigma}=0$. If $\sigma_{i}^{\prime} \neq \sigma_{\psi(i)}+\sigma_{i}^{*}$ for some $i \in[n]$, then the adversary has succeeded in creating a non-trivial polynomial $f^{*}(X)=\sum_{i=1}^{n} f_{i}^{*} X^{\lambda_{i}}+f_{0}^{*}$, with $f_{i}^{*}=\sigma_{\psi(i)}+\sigma_{i}^{*}-\sigma_{i}^{\prime}$ and $f_{0}^{*}=R_{\sigma}+\sum_{i=1}^{n} \sigma_{i} r_{i}-v_{\sigma}$, such that $f^{*}(x)=0$. By using an efficient polynomial factorization algorithm, one can now find all $\lambda_{n}+1$ roots of $f^{*}(X)$. For one of those roots, say $y$, we have $g_{2}^{y}=g_{2}^{x}$. $\mathcal{A}^{\prime}$ can now return $y=x$. Since (gk, crs) only contains $f^{x^{\ell}}$ for $\ell=0$, the adversary has thus broken the $\emptyset$-PSDL assumption, an assumption that is true unconditionally since the adversary's input does not depend on $x$ at all. Thus, $\sigma_{i}^{\prime}=\sigma_{\psi(i)}+\sigma_{i}^{*}$ for $i \in[n]$.

Analogously, by the verification in step $5 \mathrm{~b}, \sum_{i=1}^{n}\left(\tau_{\psi(i)}+\tau_{i}^{*}-\tau_{i}^{\prime}\right) x^{\lambda_{i}}+R_{\tau}+\sum_{i=1}^{n} \tau_{i} r_{i}-v_{\tau}=0$, and thus, $\tau_{i}^{\prime}=\tau_{\psi(i)}+\tau_{i}^{*}$ for all $i \in[n]$. 
Finally, by the verification in step $5 \mathrm{c}$,

$$
\begin{aligned}
\hat{e}\left(\mathfrak{u}_{\mu}, g_{2}\right)= & \hat{e}\left(g_{1}, c_{\sigma} c_{\tau}\right) \cdot \prod_{i=1}^{n} \hat{e}\left(\mathfrak{u}_{i 3}, c_{2 i}\right) / \prod_{i=1}^{n} \hat{e}\left(\mathfrak{u}_{i 3}^{\prime}, g_{2, \lambda_{i}}\right) \\
= & \hat{e}\left(g_{1}, g_{2}^{R_{\sigma}+R_{\tau}+\sum_{i=1}^{n}\left(\sigma_{i}^{*}+\tau_{i}^{*}\right) x^{\lambda_{i}}}\right) \cdot \\
& \prod_{i=1}^{n} \hat{e}\left(g_{1}^{\mu_{i}+\sigma_{i}+\tau_{i}}, \exp \left(g_{2}, r_{i}+x^{\lambda_{\psi}-1(i)}\right)\right) / \prod_{i=1}^{n} \hat{e}\left(g_{1}^{\mu_{i}^{\prime}+\sigma_{i}^{\prime}+\tau_{i}^{\prime}}, g_{2}^{x^{\lambda_{i}}}\right) .
\end{aligned}
$$

Thus,

$$
\begin{aligned}
\log _{g_{1}} \mathfrak{u}_{\mu} & =R_{\sigma}+R_{\tau}+\sum_{i=1}^{n}\left(\sigma_{i}^{*}+\tau_{i}^{*}\right) x^{\lambda_{i}}+\sum_{i=1}^{n}\left(\mu_{i}+\sigma_{i}+\tau_{i}\right)\left(r_{i}+x^{\lambda_{\psi^{-1}(i)}}\right)-\sum_{i=1}^{n}\left(\mu_{i}^{\prime}+\sigma_{i}^{\prime}+\tau_{i}^{\prime}\right) x^{\lambda_{i}} \\
& =R_{\sigma}+R_{\tau}+\sum_{i=1}^{n}\left(\mu_{i}+\sigma_{i}+\tau_{i}\right) r_{i}+\sum_{i=1}^{n}\left(\mu_{\psi(i)}-\mu_{i}^{\prime}+\sigma_{\psi(i)}+\sigma_{i}^{*}-\sigma_{i}^{\prime}+\tau_{\psi(i)}+\tau_{i}^{*}-\tau_{i}^{\prime}\right) x^{\lambda_{i}} \\
& =R_{\sigma}+R_{\tau}+\sum_{i=1}^{n}\left(\mu_{i}+\sigma_{i}+\tau_{i}\right) r_{i}+\sum_{i=1}^{n}\left(\mu_{\psi(i)}-\mu_{i}^{\prime}\right) x^{\lambda_{i}}
\end{aligned}
$$

If $\mu_{i}^{\prime} \neq \mu_{\psi(i)}$ for some $i \in[n]$, then the adversary has succeeded in creating a non-trivial polynomial $f^{*}(X)=\sum_{i=1}^{n} f_{i}^{*} X^{\lambda_{i}}+f_{0}^{*}$, with $f_{i}^{*}=\sum_{i=1}^{n}\left(\mu_{\psi(i)}-\mu_{i}^{\prime}\right)$ and $f_{0}^{*}=R_{\sigma}+R_{\tau}+\sum_{i=1}^{n}\left(\mu_{i}+\sigma_{i}+\tau_{i}\right) r_{i}-v_{\mu}$, such that $f^{*}(x)=0$. By using an efficient polynomial factorization algorithm, one can now find all $\lambda_{n}+1$ roots of $f^{*}$. For one of those roots, say $y$, we have $g_{2}^{y}=g_{2}^{x}$. Since (gk, crs) only contains $g_{1}^{x^{\ell}}$ for $\ell \in \Lambda$, the adversary has thus broken the $\Lambda$-PSDL assumption. Therefore, due to the $\Lambda$-PSDL assumption, $\mu_{i}^{\prime}=\mu_{\psi(i)}$ for $\left.i \in[n]\right]^{3}$

Thus, $\mathfrak{u}_{i 1}^{\prime}=f^{\sigma_{\psi(i)}+\sigma_{i}^{*}}, \mathfrak{u}_{i 2}^{\prime}=h^{\tau_{\psi(i)}+\tau_{i}^{*}}, \mathfrak{u}_{i 3}^{\prime}=g_{1}^{\mu_{\psi(i)}+\sigma_{\psi(i)}+\sigma_{i}^{*}+\tau_{\psi(i)}+\tau_{i}^{*}}$ and similarly for elements $\tilde{\mathfrak{u}}_{i j}^{\prime}$, and therefore, $\left\{\mathfrak{u}_{i}^{\prime}\right\}$ is indeed a correct shuffle of $\left\{\mathfrak{u}_{i}\right\}$.

PerfeCt ZERO-KNOWLEDGe: We construct a simulator $\mathcal{S}=\left(\mathcal{S}_{1}, \mathcal{S}_{2}\right)$ as follows. First, $\mathcal{S}_{1}$ generates random $\stackrel{\circ}{a}, \bar{\alpha}, x \leftarrow \mathbb{Z}_{q}$, and sets $\mathrm{td} \leftarrow(\stackrel{a}{a}, \bar{\alpha}, x)$. He then creates crs as in Prot. 4, and stores td. The construction of $\mathcal{S}_{2}$ is given in Prot. 5. Next, we give an analysis of the simulated proof. Note that $c_{\sigma}$, $c_{\tau}$ and $c_{2 i}$ are independent and random variables in $\mathbb{G}$, exactly as in the real run of the protocol. With respect to those variables, we define $\mathfrak{u}_{\sigma}, \mathfrak{u}_{\tau}$ and $\mathfrak{u}_{\mu}$ so that they satisfy the verification equations. Thus, we are now only left to show that the verification equations in steps $5 \mathrm{a}, 5 \mathrm{~b}$ and $5 \mathrm{c}$ hold.

Clearly, $\pi^{p m}$ is simulated correctly, since $\hat{e}\left(\stackrel{\circ}{g}_{1},\left(\prod_{i=1}^{n} c_{2 i}\right) / D\right)=\hat{e}\left(g_{1}, \pi^{0}\right), \hat{e}\left(c_{1 i}, g_{2}\right)=\hat{e}\left(g_{1}, c_{2 i}\right)$, $\hat{e}\left(\bar{c}_{1 i}, g_{2}\right)=\hat{e}\left(c_{1 i}, \bar{g}_{2}\right), \hat{e}\left(g_{1}, \bar{c}_{2 i}\right)=\hat{e}\left(\bar{g}_{1}, c_{2 i}\right), \hat{e}\left(g_{1}, \bar{F}_{i}\right)=\hat{e}\left(\bar{g}_{1}, F_{i}\right)$, and $\hat{e}\left(c_{1 i}, c_{2 i}\right)=\hat{e}\left(g_{1}^{z_{i}}, g_{2}^{z_{i}}\right)=$ $\hat{e}\left(g_{1}, g_{2}^{z_{i}^{2}}\right)=\hat{e}\left(g_{1}, F_{i}\right)$.

Finally, we have

$$
\begin{aligned}
\hat{e}\left(f, c_{\sigma}\right) \cdot \prod_{i=1}^{n} \hat{e}\left(\mathfrak{u}_{i 1}, c_{2 i}\right) & =\hat{e}\left(f, \prod_{i=1}^{n} g_{2}^{r_{i 1}}\right) \cdot \prod_{i=1}^{n} \hat{e}\left(\mathfrak{u}_{i 1}, g_{2}^{z_{i}}\right)=\hat{e}\left(\prod_{i=1}^{n} f^{r_{i 1}} \cdot \prod_{i=1}^{n} \mathfrak{u}_{i 1}^{z_{i}}, g_{2}\right) \\
& =\hat{e}\left(\prod_{i=1}^{n}\left(f^{r_{i 1}} \mathfrak{u}_{i 1}^{z_{i}}\left(\mathfrak{u}_{i 1}^{\prime}\right)^{-x^{\lambda_{i}}}\right), g_{2}\right) \cdot \prod_{i=1}^{n} \hat{e}\left(\mathfrak{u}_{i 1}^{\prime}, g_{2, \lambda_{i}}\right)=\hat{e}\left(\mathfrak{u}_{\sigma}, g_{2}\right) \cdot \prod_{i=1}^{n} \hat{e}\left(\mathfrak{u}_{i 1}^{\prime}, g_{2, \lambda_{i}}\right) .
\end{aligned}
$$

Similarly, $\hat{e}\left(h, c_{\tau}\right) \cdot \prod_{i=1}^{n} \hat{e}\left(\mathfrak{u}_{i 2}, c_{2 i}\right)=\hat{e}\left(\mathfrak{u}_{\tau}, g_{2}\right) \cdot \prod_{i=1}^{n} \hat{e}\left(\mathfrak{u}_{i 2}^{\prime}, g_{2, \lambda_{i}}\right)$ and $\hat{e}\left(g_{1}, c_{\sigma} c_{\tau}\right) \cdot \prod_{i=1}^{n} \hat{e}\left(\mathfrak{u}_{i 3}, c_{2 i}\right)=$ $\hat{e}\left(\mathfrak{u}_{\mu}, g_{2}\right) \cdot \prod_{i=1}^{n} \hat{e}\left(\mathfrak{u}_{i 3}^{\prime}, g_{2, \lambda_{i}}\right)$. Thus all three verification equations hold, and therefore the simulator has succeeded in generating an argument that has the same distribution as the real argument.

Theorem 6. Consider Prot. 4. The CRS consists of $2 n+2$ elements of $\mathbb{G}_{1}$ and $5 n+4$ elements of $\mathbb{G}_{2}$, in total $7 n+6$ group elements. The communication complexity is $2 n+6$ elements of $\mathbb{G}_{1}$ and $4 n+5$ elements of $\mathbb{G}_{2}$, in total $6 n+11$ group elements. The prover's computational complexity is dominated by $17 n+16$ exponentiations. The verifier's computational complexity is dominated by $28 n+18$ pairings.

\footnotetext{
${ }^{3}$ For the argument in this paragraph to go through, we need the knowledge BBS cryptosystem to be lifted and the plaintexts to be small. Otherwise, the adversary will not know the coefficients of $f^{\prime}(X)$, and thus one could not use a polynomial factorization algorithm to break the $\Lambda$-PSDL assumption. Thus, a crafty adversary might be able to break soundness by choosing $g_{1}^{\mu}$ from which she cannot compute $\mu$.
} 


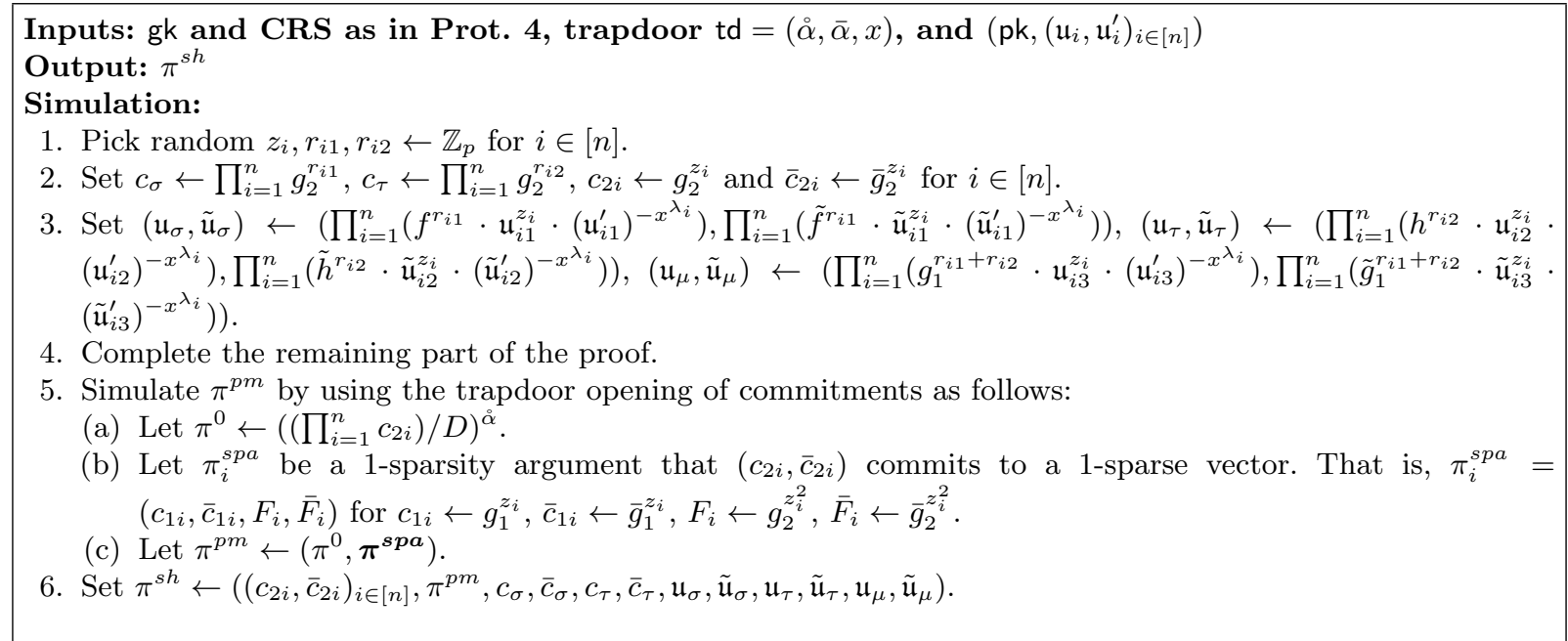

Protocol 5: Simulator $\mathcal{S}_{2}$ : construction

Proof. The communication complexity: $\left|\pi^{p m}\right|$; in addition, 6 elements from $\mathbb{G}_{1}$ and $2 n+4$ elements from $\mathbb{G}_{2}$. The prover's computational complexity follows from that of the permutation matrix argument, to which the shuffle argument proper adds $7 n+15$ exponentiations. Finally, the shuffle argument proper adds $18 n+16$ bilinear pairings to the verifier's computational complexity of the permutation matrix argument. The rest is straightforward.

We note that in a mix server-like application where several shuffles are done sequentially, one can get somewhat smaller amortized cost. Namely, the output ciphertext $\mathfrak{u}_{i}^{\prime}$ of one shuffle is equal to the input ciphertext $\mathfrak{u}_{i}$ of the following shuffle. Therefore, in step 3 , one only has to check the correctness of the ciphertexts $\mathfrak{u}_{i}^{\prime}$ in the case of the very last shuffle. This means that the verifier's amortized computational complexity is dominated by $22 n+18$ pairings (that is, one has thus saved $6 n$ pairings).

Acknowledgments. We would like to thank Jens Groth for insightful comments. The work was done while the second author was working at the University of Tartu. The authors were supported by Estonian Science Foundation, grant \#9303, and European Union through the European Regional Development Fund.

\section{References}

AF07. Masayuki Abe and Serge Fehr. Perfect NIZK with Adaptive Soundness. In Salil Vadhan, editor, TCC 2007, volume 4392 of LNCS, pages 118-136, Amsterdam, The Netherlands, February 21-24, 2007. Springer, Heidelberg.

BBS04. Dan Boneh, Xavier Boyen, and Hovav Shacham. Short Group Signatures. In Matthew K. Franklin, editor, CRYPTO 2004, volume 3152 of LNCS, pages 41-55, Santa Barbara, USA, August 15-19, 2004. Springer, Heidelberg.

BG12. Stephanie Bayer and Jens Groth. Efficient Zero-Knowledge Argument for Correctness of a Shuffle. In David Pointcheval and Thomas Johansson, editors, EUROCRYPT 2012, volume 7237 of LNCS, pages 263-280, Cambridge, UK, April 15-19, 2012. Springer, Heidelberg.

BN05. Paulo S. L. M. Barreto and Michael Naehrig. Pairing-Friendly Elliptic Curves of Prime Order. In Bart Preneel and Stafford E. Tavares, editors, SAC 2005, volume 3897 of LNCS, pages 319-331, Kingston, ON, Canada, August 11-12, 2005. Springer, Heidelberg.

Bou00. Fabrice Boudot. Efficient Proofs That a Committed Number Lies in an Interval. In Bart Preneel, editor, EUROCRYPT 2000, volume 1807 of LNCS, pages 431-444, Bruges, Belgium, May 14-18, 2000. Springer, Heidelberg.

BP04. Mihir Bellare and Adriana Palacio. Towards Plaintext-Aware Public-Key Encryption without Random Oracles. In Pil Joong Lee, editor, ASIACRYPT 2004, volume 3329 of LNCS, pages 48-62, Jeju Island, Korea, December 5-9 2004. Springer, Heidelberg. 
CCs08. Jan Camenisch, Rafik Chaabouni, and abhi shelat. Efficient Protocols for Set Membership and Range Proofs. In Josef Pieprzyk, editor, ASIACRYPT 2008, volume 5350 of LNCS, pages 234-252, Melbourne, Australia, December 7-11, 2008. Springer, Heidelberg.

CGH98. Ran Canetti, Oded Goldreich, and Shai Halevi. The Random Oracle Methodology, Revisited. In Jeffrey Scott Vitter, editor, STOC 1998, pages 209-218, Dallas, Texas, USA, May 23-26, 1998.

CGS97. Ronald Cramer, Rosario Gennaro, and Berry Schoenmakers. A Secure and Optimally Efficient MultiAuthority Election Scheme. In Walter Fumy, editor, EUROCRYPT 1997, volume 1233 of LNCS, pages 103-118, Konstanz, Germany, 11-15 May 1997. Springer, Heidelberg.

Cha81. David Chaum. Untraceable Electronic Mail, Return Addresses, and Digital Pseudonyms. Communications of the ACM, 24(2):84-88, 1981.

Clo53. Charles Clos. A Study of Non-Blocking Switching Networks. Bell System Technical Journal, 32(2):406424, March 1953.

CLs10. Rafik Chaabouni, Helger Lipmaa, and abhi shelat. Additive Combinatorics and Discrete Logarithm Based Range Protocols. In Ron Steinfeld and Philip Hawkes, editors, ACISP 2010, volume 6168 of LNCS, pages 336-351, Sydney, Australia, July 5-7, 2010. Springer, Heidelberg.

CLZ12. Rafik Chaabouni, Helger Lipmaa, and Bingsheng Zhang. A Non-Interactive Range Proof with Constant Communication. In Angelos Keromytis, editor, FC 2012, volume ? of LNCS, pages ?-?, Bonaire, The Netherlands, February 27-March 2, 2012. Springer, Heidelberg.

Dam91. Ivan Damgård. Towards Practical Public Key Systems Secure against Chosen Ciphertext Attacks. In Joan Feigenbaum, editor, CRYPTO 1991, volume 576 of LNCS, pages 445-456, Santa Barbara, California, USA, August 11-15, 1991. Springer, Heidelberg, 1992.

DT04. William James Dally and Brian Patrick Towles. Principles and Practices of Interconnection Networks. Morgan Kaufmann, 2004.

Elk11. Michael Elkin. An Improved Construction of Progression-Free Sets. Israeli Journal of Mathematics, 184:93-128, 2011.

ET36. Paul Erdős and Paul Turán. On Some Sequences of Integers. Journal of the London Mathematical Society, 11(4):261-263, 1936.

FS86. Amos Fiat and Adi Shamir. How to Prove Yourself: Practical Solutions to Identification and Signature Problems. In Andrew M. Odlyzko, editor, CRYPTO 1986, volume 263 of LNCS, pages 186-194, Santa Barbara, California, USA, 11-15 August 1986. Springer, Heidelberg, 1987.

FS01. Jun Furukawa and Kazue Sako. An Efficient Scheme for Proving a Shuffle. In Joe Kilian, editor, CRYPTO 2001, volume 2139 of LNCS, pages 368-387, Santa Barbara, USA, August 19-23, 2001. Springer, Heidelberg.

GI08. Jens Groth and Yuval Ishai. Sub-linear Zero-Knowledge Argument for Correctness of a Shuffle. In Nigel Smart, editor, EUROCRYPT 2008, volume 4965 of LNCS, pages 379-396, Istanbul, Turkey, April 13-17, 2008. Springer, Heidelberg.

GJM02. Philippe Golle, Stanislaw Jarecki, and Ilya Mironov. Cryptographic Primitives Enforcing Communication and Storage Complexity. In Matt Blaze, editor, FC 2002, volume 2357 of LNCS, pages 120-135, Southhampton Beach, Bermuda, March 11-14, 2002. Springer, Heidelberg.

GK03. Shafi Goldwasser and Yael Tauman Kalai. On the (In)security of the Fiat-Shamir Paradigm. In FOCS 2003, pages 102-113, Cambridge, MA, USA, October, 11-14 2003. IEEE, IEEE Computer Society Press.

GL07. Jens Groth and Steve Lu. A Non-interactive Shuffle with Pairing Based Verifiability. In Kaoru Kurosawa, editor, ASIACRYPT 200\%, volume 4833 of LNCS, pages 51-67, Kuching, Malaysia, December 26, 2007. Springer, Heidelberg.

GMR85. Shafi Goldwasser, Silvio Micali, and Charles Rackoff. The Knowledge Complexity of Interactive ProofSystems. In Robert Sedgewick, editor, STOC 1985, pages 291-304, Providence, Rhode Island, USA, May 6-8, 1985. ACM Press.

GOS06. Jens Groth, Rafail Ostrovsky, and Amit Sahai. Perfect Non-Interactive Zero-Knowledge for NP. In Serge Vaudenay, editor, EUROCRYPT 2006, volume 4004 of LNCS, pages 338-359, St. Petersburg, Russia, May 28-June 1, 2006. Springer, Heidelberg.

GOS11. Jens Groth, Rafail Ostrovsky, and Amit Sahai. New Techniques for Non-interactive Zero Knowledge. Full version of GOS06]. Draft, available from the authors, March 7, 2011.

Gro03. Jens Groth. A Verifiable Secret Shuffle of Homomorphic Encryptions. In Yvo Desmedt, editor, $P K C$ 2003, volume 2567 of $L N C S$, pages 145-160, Miami, Florida, USA, January 6-8, 2003. Springer, Heidelberg.

Gro09. Jens Groth. Linear Algebra with Sub-linear Zero-Knowledge Arguments. In Shai Halevi, editor, CRYPTO 2009, volume 5677 of LNCS, pages 192-208, Santa Barbara, California, USA, August 16-20, 2009. Springer, Heidelberg.

Gro10. Jens Groth. Short Pairing-Based Non-interactive Zero-Knowledge Arguments. In Masayuki Abe, editor, ASIACRYPT 2010, volume 6477 of LNCS, pages 321-340, Singapore, December 5-9 2010. Springer, Heidelberg. 
GS08. Jens Groth and Amit Sahai. Efficient Non-interactive Proof Systems for Bilinear Groups. In Nigel Smart, editor, EUROCRYPT 2008, volume 4965 of LNCS, pages 415-432, Istanbul, Turkey, April 1317, 2008. Springer, Heidelberg.

HSV06. Florian Hess, Nigel P. Smart, and Frederik Vercauteren. The Eta Pairing Revisited. IEEE Transactions on Information Theory, 52(10):4595-4602, 2006.

KNP10. Kaoru Kurosawa, Ryo Nojima, and Le Trieu Phong. Efficiency-Improved Fully Simulatable Adaptive OT under the DDH Assumption. In Juan Garay, editor, SCN 2010, volume 6280 of LNCS, pages 172-181, Amalfi, Italy, September 13-15, 2010. Springer Verlag.

KNP11. Kaoru Kurosawa, Ryo Nojima, and Le Trieu Phong. Generic Fully Simulatable Adaptive Oblivious Transfer. In Javier Lopez and Gene Tsudik, editors, ACNS 2011, volume 6715 of LNCS, pages 274-291, Nerja, Spain, June 7-10, 2011. Springer, Heidelberg.

LAN02. Helger Lipmaa, N. Asokan, and Valtteri Niemi. Secure Vickrey Auctions without Threshold Trust. In Matt Blaze, editor, FC 2002, volume 2357 of LNCS, pages 87-101, Southhampton Beach, Bermuda, March 11-14, 2002. Springer, Heidelberg.

Lip03. Helger Lipmaa. On Diophantine Complexity and Statistical Zero-Knowledge Arguments. In Chi Sung Laih, editor, ASIACRYPT 2003, volume 2894 of LNCS, pages 398-415, Taipei, Taiwan, November 30-December 4, 2003. Springer, Heidelberg.

Lip12. Helger Lipmaa. Progression-Free Sets and Sublinear Pairing-Based Non-Interactive Zero-Knowledge Arguments. In Ronald Cramer, editor, TCC 2012, volume 7194 of LNCS, pages 169-189, Taormina, Italy, March 18-21, 2012. Springer, Heidelberg.

LZ12. Helger Lipmaa and Bingsheng Zhang. A More Efficient Computationally Sound Non-Interactive ZeroKnowledge Shuffle Argument. In Ivan Visconti, editor, SCN 2012, volume ? of LNCS, pages ?-?, Amalfi, Italy, September 5-7, 2012. Springer, Heidelberg.

Nef01. C. Andrew Neff. A Verifiable Secret Shuffle and Its Application to E-Voting. In ACM CCS 2001, pages 116-125, Philadelphia, Pennsylvania, USA, November 6-8 2001. ACM Press.

PSNB11. C. C. F. Pereira Geovandro, Marcos A. Simplício Jr., Michael Naehrig, and Paulo S. L. M. Barreto. A Family of Implementation-Friendly BN Elliptic Curves. Journal of Systems and Software, 84(8):13191326, 2011.

RKP09. Alfredo Rial, Markulf Kohlweiss, and Bart Preneel. Universally Composable Adaptive Priced Oblivious Transfer. In Hovav Shacham and Brent Waters, editors, Pairing 2009, volume 5671 of LNCS, pages 231-247, Palo Alto, CA, USA, August 12-14, 2009. Springer, Heidelberg.

San11. Tom Sanders. On Roth's Theorem on Progressions. Annals of Mathematics, 174(1):619-636, July 2011.

Sch80. Jacob T. Schwartz. Fast Probabilistic Algorithms for Verification of Polynomial Identities. Journal of the ACM, 27(4):701-717, 1980.

SV12. Alessandra Scafuro and Ivan Visconti. On Round-Optimal Zero Knowledge in the Bare Public-Key Model. In David Pointcheval and Thomas Johansson, editors, EUROCRYPT 2012, volume 7237 of LNCS, pages 153-171, Cambridge, UK, April 15-19, 2012. Springer, Heidelberg.

TV06. Terrence Tao and Van Vu. Additive Combinatorics. Cambridge Studies in Advanced Mathematics. Cambridge University Press, 2006.

TW10. Björn Terelius and Douglas Wikström. Proofs of Restricted Shuffles. In Daniel J. Bernstein and Tanja Lange, editors, AFRICACRYPT 2010, volume 6055 of LNCS, pages 100-113, Stellenbosch, South Africa, May 3-6, 2010. Springer, Heidelberg.

vHN10. Mark van Hoeij and Andrew Novocin. Gradual Sub-lattice Reduction and a New Complexity for Factoring Polynomials. In Alejandro López-Ortiz, editor, LATIN 2010, volume 6034 of LNCS, pages 539-553, Oaxaca, Mexico, April 19-23, 2010. Springer, Heidelberg.

\section{A Decisional Linear Assumption}

We say that a bilinear group generator $\mathcal{G}_{\mathrm{bp}}$ is DLIN (decisional linear) secure BBS04 in group $\mathbb{G}_{t}$, for $t \in\{1,2\}$, if for all non-uniform polynomial time adversaries $\mathcal{A}$, the following probability is negligible in $\kappa$ :

$$
\operatorname{Pr}\left[\begin{array}{l}
\mathrm{gk} \leftarrow \mathcal{G}_{\mathrm{bp}}\left(1^{\kappa}\right), \\
(f, h) \leftarrow\left(\mathbb{G}_{t}^{*}\right)^{2},(\sigma, \tau) \leftarrow \mathbb{Z}_{p}^{2}: \\
\mathcal{A}\left(\mathrm{gk} ; f, h, f^{\sigma}, h^{\tau}, g_{t}^{\sigma+\tau}\right)=1
\end{array}\right]-\operatorname{Pr}\left[\begin{array}{l}
\mathrm{gk} \leftarrow \mathcal{G}_{\mathrm{bp}}\left(1^{\kappa}\right), \\
(f, h) \leftarrow\left(\mathbb{G}_{t}^{*}\right)^{2},(\sigma, \tau, z) \leftarrow \mathbb{Z}_{p}^{3}: \\
\mathcal{A}\left(\mathrm{gk} ; f, h, f^{\sigma}, h^{\tau}, g_{t}^{z}\right)=1
\end{array}\right] \mid .
$$

\section{B Groth-Lu Co-Soundness Definition}

The Groth-Lu shuffle argument is proven to be $R_{c o}^{s h}$-sound with respect to the next language [GL07] (here, as in GL07, we assume the setting of symmetric pairings $\hat{e}: \mathbb{G} \times \mathbb{G} \rightarrow \mathbb{G}_{T}$, and like GL07] we 
give the definition with respect to the BBS cryptosystem only):

$$
R_{c o}^{s h}:=\left\{\begin{array}{l}
\left(\left(p, \mathbb{G}, \mathbb{G}_{T}, \hat{e}, g\right),\left(f, h,\left\{\mathfrak{u}_{i}\right\},\left\{\mathfrak{u}_{i}^{\prime}\right\}\right), \mathrm{sk}=\left(\mathrm{sk}_{1}, \mathrm{sk}_{2}\right)\right):(x, y) \in\left(\mathbb{Z}_{p}^{*}\right)^{2} \wedge \\
f=g^{\mathrm{sk}_{1}} \wedge h=g^{s \mathrm{k}_{2}} \wedge\left(\forall \psi \in S_{n} \exists i: \mathcal{D e c}_{\mathrm{sk}}\left(\mathfrak{u}_{i}^{\prime}\right) \neq \operatorname{Dec}_{\mathrm{sk}}\left(\mathfrak{u}_{\psi(i)}\right)\right)
\end{array}\right\} .
$$

That is, the adversary is required to return not only a non-shuffle $\left(\left\{\mathfrak{u}_{i}\right\},\left\{\mathfrak{u}_{i}^{\prime}\right\}\right)$, but also a secret key sk that makes it possible to verify efficiently that $\left(\left\{\mathfrak{u}_{i}\right\},\left\{\mathfrak{u}_{i}^{\prime}\right\}\right)$ is really not a shuffle. As argued in GL07, this definition of $R_{c o}^{s h}$ makes sense in practice, since there is always some coalition of the parties who knows the secret key. See GL07] for more.

\section{PPA Assumption from [GL07]}

Mostly for comparison reasons, we will state next the definition of the permutation pairing assumption from [GL07. Since the original definition was given in the symmetric setting, we assume here that $\mathbb{G}=\mathbb{G}_{1}=\mathbb{G}_{2}$, and $g=g_{1}=g_{2}$.

Definition 1 (PPA Assumption [GL07]). The permutation pairing assumption holds for $\mathcal{G}_{\mathrm{bp}}$, if for all non-uniform PPT adversaries, the following probability is negligible in $\kappa$ :

$$
\operatorname{Pr}\left[\begin{array}{l}
\mathrm{gk}:=\left(p, \mathbb{G}, \mathbb{G}, \mathbb{G}_{T}, g, g\right) \leftarrow \mathcal{G}_{\mathrm{bp}}\left(1^{\kappa}\right),\left(x_{1}, \ldots, x_{n}\right) \leftarrow \mathbb{Z}_{p}^{n}, \\
\left(a_{i}, b_{i}\right)_{i \in[n]} \leftarrow \mathcal{A}\left(\mathrm{gk},\left(g^{x_{i}}, g^{x_{i}^{2}}\right)_{i \in[n]}\right): \\
\prod_{i=1}^{n} a_{i} g^{-x_{i}}=1 \wedge \prod_{i=1}^{n} b_{i} g^{-x_{i}^{2}}=1 \wedge(\forall i \in[n]) \hat{e}\left(a_{i}, a_{i}\right)=\hat{e}\left(g, b_{i}\right) \wedge \\
\left(a_{i}\right)_{i \in[n]} \text { is not a permutation of }\left(g^{x_{i}}\right)_{i \in[n]}
\end{array}\right] .
$$

\section{Shortening the CRS}

Preliminaries on Clos network. An $\left(n_{2}, n_{1}, r\right)$-Clos network Clo53|DT04 for permutation $\pi \in S_{n_{1} r}$ is a three-stage network to implement $\pi$, in which each stage is composed in a number of smaller permutations. The first stage has $r$ small permutations (input switches, each from $S_{n_{1}}$ ), the second stage has $n_{2}$ small permutations (middle stage switches, each from $S_{r}$ ), and the third stage has $r$ small permutations (output switches, each from $S_{n_{1}}$ ). Each input switch is connected to each middle stage switch, and each middle stage switch is connected to each output switch, see Fig. 1 .

To implement an arbitrary permutation from $S_{n_{1} r}$ it suffices to use a so called rearrangeably nonblocking network Clo53|DT04, for which one can choose an $\left(n_{2}=n_{1}, n_{1}, r\right)$-Clos network. For this one just has to choose the $2 r+n_{1}$ small permutations accordingly.

Shortening the CRS by using a Clos network. Consider an arbitrary permutation $\psi \in S_{n}$ for some large $n$. Instead of implementing directly a shuffle argument for $\psi$, one can instead construct an $\left(n_{1}, n_{1}, r=n / n_{1}\right)$-Clos network for $\psi$, as follows (we assume, w.l.o.g., that $n$ divides by $\left.n_{1}\right)$ :

1. Divide $n$ input ciphertexts $\mathfrak{u}_{i}, i \in[n]$, between $n / n_{1}$ input switches, where each input switch implements a permutation on $n_{1}$ elements.

2. Construct a shuffle argument for each input switch. That is, each input switch outputs a permuted and rerandomized list of its $n_{1}$ input ciphertexts together with a corresponding shuffle argument $\pi_{i n p: i}^{s h}, i \in\left[n / n_{1}\right]$.

3. The $n$ output ciphertexts $\mathfrak{u}_{i}^{\prime}$ of the first stage are sent to the middle stage switches according to the Clos network connections.

4. Construct a shuffle argument for each middle stage switch. That is, each middle stage switch outputs a permuted and rerandomized list of its $n / n_{1}$ input ciphertexts together with a corresponding shuffle argument $\pi_{\text {mid: } i}^{\text {sh }}, i \in\left[n_{1}\right]$.

5. The $n$ output ciphertexts $\mathfrak{u}_{i}^{\prime \prime}$ of the middle stage are sent to the output switches according to the Clos network connections. 


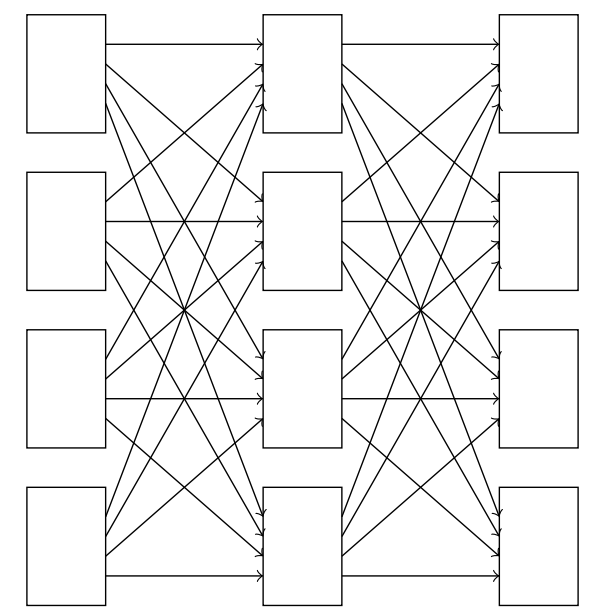

Fig. 1. A $(4,4,4)$-Clos network for implementing any permutation from $S_{16}$

6. Construct a shuffle argument for each output switch. That is, each output switch outputs a permuted and rerandomized list of its $n_{1}$ input ciphertexts together with a corresponding shuffle argument $\pi_{\text {out }: i}^{\text {sh }}, i \in\left[n / n_{1}\right]$.

7. Let $\mathfrak{u}_{i}^{\prime \prime \prime}$ be the $n$ output ciphertexts of the third stage.

The whole shuffle argument for $\psi$ is equal to

$$
\pi^{s h}=\left(\left\{\mathfrak{u}_{i}^{\prime}: i \in[n]\right\},\left\{\mathfrak{u}_{i}^{\prime \prime}: i \in[n]\right\},\left\{\pi_{\text {inp:i }}^{s h}: i \in\left[n / n_{1}\right]\right\},\left\{\pi_{\text {mid:i }}^{s h}: i \in\left[n_{1}\right]\right\},\left\{\pi_{\text {out:i }}^{s h}: i \in\left[n_{1}\right]\right\}\right)
$$

(note that $\mathfrak{u}_{i}^{\prime \prime \prime}$ are the output ciphertexts and thus not formally part of the shuffle argument itself).

Now, assuming that the length of the CRS, prover's computation, verifier's computation and the argument size in the case of a shuffle argument on $n^{\prime}$ elements are respectively $c l\left(n^{\prime}\right), p c\left(n^{\prime}\right), v c\left(n^{\prime}\right)$, and $\operatorname{com}\left(n^{\prime}\right)$ in corresponding units respectively, and that ciphertext length is $u l$ units, we get that $\operatorname{com}(n)=2 n \cdot u l+2 n / n_{1} \cdot \operatorname{com}\left(n_{1}\right)+n_{1} \cdot \operatorname{com}\left(n / n_{1}\right)$. This is clearly minimized when $n_{1}=\sqrt{n}$, in which case $\operatorname{com}(n)=2 n \cdot u l+3 \sqrt{n} \cdot \operatorname{com}(\sqrt{n})$. In this case, $p c(n)$ is dominated by $3 \sqrt{n} \cdot p c(\sqrt{n})$ (plus $2 \cdot 6 n=12 n$ exponentiations to form intermediate ciphertexts), and $v c(n)$ is dominated by $3 \sqrt{n} \cdot v c(\sqrt{n})$.

Since, according to Tbl. 1, in the case of the shuffle of this paper, $\operatorname{com}(\sqrt{n})=6 \sqrt{n}+11, p c(\sqrt{n})=$ $17 \sqrt{n}+16$ and $v c(\sqrt{n})=28 \sqrt{n}+18$ are linear (in $\sqrt{n}$ ), we get that the Clos-networked version of the shuffle argument has $\operatorname{com}(n)=2 \cdot 6 n+3 \sqrt{n} \cdot(6 \sqrt{n}+11)=30 n+33 \sqrt{n}, p c(n)=12 n+3 \sqrt{n} \cdot(17 \sqrt{n}+$ 16) $=63 n+48 \sqrt{n}, v c(n)=3 \sqrt{n} \cdot(28 \sqrt{n}+18)=84 n+54 \sqrt{n}$. Therefore, all those parameters become approximately 3 to 5 times more expensive. On the other hand, all small permutations can share the same CRS of length $7 \sqrt{n}+6$ of group elements, and therefore the Clos-networked version of the new shuffle arguments has quadratically shorter CRS.

Applying Clos networks recursively $t$ times (where $t$ can but does not have to be a constant), we arrive to a shuffle argument where the communication and computation are dominated by $\Theta\left(c^{t} n\right)$ (for $c \in[3,5])$ while the CRS length is $n^{1 / 2^{t}}$. One can alternatively apply the Beneš network DT04 to reduce the CRS to a constant while increasing the computation or communication to $\Theta(n \log n)$.

The same techniques can also clearly be applied to the Groth-Lu argument. 